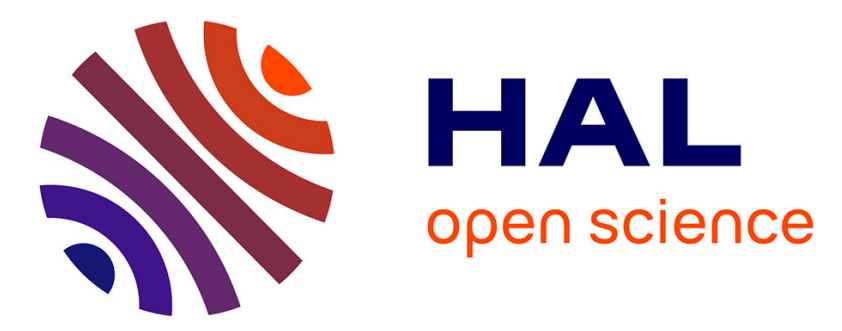

\title{
First measurements of Jupiter's zonal winds with visible imaging spectroscopy
}

\author{
Ivan Gonçalves, Schmider F.X., Patrick Gaulme, Raúl Morales-Juberías, \\ Tristan Guillot, Jean-Pierre Rivet, Thierry Appourchaux, Patrick Boumier, \\ Jason Jackiewicz, Bun'ei Sato, et al.
}

\section{To cite this version:}

Ivan Gonçalves, Schmider F.X., Patrick Gaulme, Raúl Morales-Juberías, Tristan Guillot, et al.. First measurements of Jupiter's zonal winds with visible imaging spectroscopy. Icarus, 2019, 319, pp.795811. 10.1016/j.icarus.2018.10.019 . hal-02105121

\section{HAL Id: hal-02105121 \\ https://hal.science/hal-02105121}

Submitted on 14 Jan 2021

HAL is a multi-disciplinary open access archive for the deposit and dissemination of scientific research documents, whether they are published or not. The documents may come from teaching and research institutions in France or abroad, or from public or private research centers.
L'archive ouverte pluridisciplinaire HAL, est destinée au dépôt et à la diffusion de documents scientifiques de niveau recherche, publiés ou non, émanant des établissements d'enseignement et de recherche français ou étrangers, des laboratoires publics ou privés. 


\title{
First measurements of Jupiter's zonal winds with visible imaging spectroscopy
}

Ivan Gonçalves $^{\mathrm{a}}$, F.X Schmider ${ }^{\mathrm{a}}$, Patrick Gaulme ${ }^{\mathrm{b}, \mathrm{c}, \mathrm{d}}$, Raúl Morales-Juberías ${ }^{\mathrm{d}}$, Tristan Guillot ${ }^{\mathrm{a}}$, Jean-Pierre Rivet ${ }^{\mathrm{a}}$, Thierry Appourchaux $^{\mathrm{e}}$, Patrick Boumiere ${ }^{\mathrm{e}}$, Jason Jackiewicz ${ }^{\mathrm{c}}$, Bun'ei Sato ${ }^{\mathrm{f}}$, Shigeru Ida ${ }^{\mathrm{f}}$, Masahiro Ikoma ${ }^{\mathrm{g}}$, Djamel Mékarnia ${ }^{\mathrm{a}}$, Thomas A Underwood $^{\text {h }}$, David Voelz ${ }^{\mathrm{h}}$

\author{
${ }^{a}$ Laboratoire Lagrange, Université Côte d'Azur, UMR 7293, Observatoire de la Côte d'Azur (OCA), Nice, France \\ ${ }^{b}$ Max-Planck-Institut für Sonnensystemforschung, Justus-von-Liebig-Weg 3, 37077, Göttingen, Germany \\ ${ }^{c}$ Department of Astronomy, New Mexico State University, P.O. Box 30001, MSC 4500, Las Cruces, NM 88003-8001, USA \\ ${ }^{d}$ Physics Department, New Mexico Institute of Mining and Technology, 801 Leroy Place, Socorro, NM 87801, USA \\ e Institut d'Astrophysique Spatiale, Université Paris Sud, 91405 Orsay Cedex, France \\ ${ }^{f}$ Tokyo Institut of Technology, Dept. of Earth and Planetary Sciences, 2-12-1 Ookayama, Meguro, Tokyo 152-8551, Japan \\ ${ }^{g}$ Department of Earth and Planetary Science, The University of Tokyo, 7-3-1 Hongo Bunkyo-ku, Tokyo 113-0033, Japan \\ ${ }^{h}$ Klipsch School of Electrical and Computer Engineering, New Mexico State University, MSC 3-O, Goddard Annex 160B, Las Cruces, NM 88003, USA
}

\begin{abstract}
We present the first measurements of Jupiter's wind profile ever obtained with Doppler velocity measurements in the visible. Hitherto, knowledge about atmospheric dynamics has been obtained with cloud-tracking techniques, which consist of tracking visible features from images taken at different dates. However, cloud tracking indicates the motion of large cloud structures, which is an indication of the speed of iso-pressure regions, rather than the speed of the actual atmospheric particles. Doppler imaging is as challenging - motions are usually less than $100 \mathrm{~m} \mathrm{~s}^{-1}$ - as appealing because it measures the speed of cloud particles instead of large cloud structures. Significant difference could appear in the case of atmospheric waves interfering with cloud structures. Here we present the first scientific results of a Doppler imaging spectrometer that is dedicated to giant-planet seismology and atmospheric dynamics by providing instantaneous line-of-sight-velocity maps of the planets of the solar system. The instrument has been developed in the framework of the projects JOVIAL (Jovian Oscillations through Velocity Images At several Longitudes) and JIVE in NM (Jovian Interiors from Velocimetry Experiment in New Mexico). It is a Fourier transform spectrometer with a fixed optical path difference working in the mid-visible domain, which monitors the position of solar Fraunhofer lines that are reflected in the planets' upper atmospheres. After describing the instrument principle and the different steps of data reduction, we report measurement of the average zonal wind speed of Jupiter, as a function of latitude, from datasets obtained in 2015 and 2016 with two different telescopes, when the planet was close to its opposition. Our results are consistent between the two years. We compare the results with wind profiles obtained by cloud tracking on HST (Hubble Space Telescope) images taken at the same epoch, and identify a significant discrepancy in the northern equatorial band.
\end{abstract}

Keywords: Atmospheres, dynamics, Instrumentation, Spectroscopy, Jupiter

\section{Context}

By tracking the motion of visible clouds in the troposphere we know that the predominant weather pattern in the Jovian atmosphere consists of a series of alternating eastward and west-

5 ward zonal jets that are remarkably steady over long time scales (Limaye, 1986, Stamp and Dowling, 1993; García-Melendo 25 and Sánchez-Lavega, 2001; Porco et al., 2003). We also know that, embedded between these alternating jets, other dynamical structures such as vortices and waves develop (Mac Low and Ingersoll, 1986, Deming et al., 1997, Morales-Juberías et al. 2002a b; Li et al., 2004; Young et al., 2005; Reuter et al., 2007, 30 Simon-Miller et al., 2012; Choi et al., 2013). Some of the most prominent of these features, like the Great Red Spot (GRS), are well characterized (Mitchell et al., 1981; Vasavada et al., 1998; Simon-Miller et al., 2002, Choi et al., 2007; Asay-Davis et al. 2009), and infrared observations indicate that Jupiter has ${ }_{35}$ a strong equatorial stratospheric jet (Flasar et al., 2004; Li et al., 2008).
A lot of information about the atmospheric dynamics have been derived from ground-based observational campaigns and from space missions like Voyager and Galileo (Ingersoll et al., 2004). However, many questions still remain unanswered. The mechanisms maintaining the dominant alternating jets, vortices and waves in the troposphere as well as their structure below the visible cloud level is largely unconstrained by the existing observations. The relationship between small-scale variability in the jets and the observed atmospheric morphology variability is also poorly understood. Finally, it remains unclear the precise role that eddies and waves play in governing Jupiter's weather pattern and its variability (both in the troposphere and in the stratosphere). Overall, the Jovian weather pattern is a complex system involving many different phenomena at different spatial and temporal scales, and we lack the continuous high-resolution observations of Jupiter's weather system as a whole needed to understand such dynamics. 
The Juno NASA spacecraft, which has been orbiting Jupiter since July 2016, aims at investigating the internal structure below the surface of the planet by measuring the gravitational mo- 90 ments. Very accurate values have been obtained so far (Bolton et al. 2017), and the accuracy keeps improving with each new flyby. However, interpreting these measurements depends on our ability to correctly model the dynamics of the surface layers, as the moments are sensitive to both local composition gra- 95 45 dients and to underlying differential rotation, which are not easy to disentangle. Thus, accurate measurements of the dynamics of Jupiter's upper atmosphere are fundamental for interpreting Juno's data.

In this paper, we present the first measurements of Jupiter's $\$ 00$ atmospheric zonal winds obtained with a new Doppler imaging spectrometer working in the visible domain, which is specially designed to provide spatially resolved line-of-sight velocities ${ }^{1}$ of the planetary atmospheres of the solar system (Gonçalves et al., 2016). This instrumental project ${ }_{105}$ 55 is supported by the Agence Nationale pour la Recherche in France under the project JOVIAL (Jovian Oscillations through Velocity Images At several Longitudes Schmider et al. 2013, and by the NASA/EPSCoR program in the United-States under the project JIVE in NM (Jovian Inte- 110 riors from Velocimetry Experiment in New Mexico Underwood et al. 2017)). We hereater refer to the whole project as "JOVIAL-JIVE".

We first discuss the complementarity of atmospheric winds measurement by Doppler velocimetry with the tra- 115 ${ }_{65}$ ditional cloud tracking methods (Sect. 2). We then present JOVIAL-JIVE's instrumental principle (Sect. 3) and we extensively describe the data processing protocol, including simulations that we employed to validate the process (Sect. 4). We then present two zonal wind profiles that we $\mathbf{e}_{120}$ obtained from observations done in 2015 and 2016, and we compare them with results obtained at the same epoch by cloud-tracking, using HST data (Sect. 5).

Beyond an innovative instrumental technique, the main scientific result of our paper is the identification of a signifi-125 75 cant discrepancy of the zonal wind profile between Doppler imaging and cloud tracking, which we observe in the north equatorial band, at the latitude of the "hot spots." These results are discussed in the last section and several hypotheses are put forth.

\section{Imaging spectroscopy versus cloud tracking}

With cloud tracking, the wind speed is derived from changes of cloud position as a function of time (Barrado-Izagirre et al. ${ }^{135}$ 2013; Tollefson et al., 2017b; Choi and Showman, 2011; Johnson et al. 2018). However, clouds are affected by traveling thermal and gravity waves, and could show an apparent displacement different from the true motion of the gas or particles. In that sense, cloud tracking is an indication of the speed ${ }^{140}$

${ }^{1}$ Note that line-of-sight velocities are also called radial velocities, which should not be confused with atmospheric vertical motions. of iso-pressure regions, rather than the speed of the actual cloud particles. Moreover, some parts of the planet are not covered by clouds, thus making cloud tracking impossible in those regions. Other methods have been used to derive the wind profile as a function of altitude using temperature measurements in the IR (Fletcher et al., 2016), but they explicitly use the cloud-tracking profile in the process.

Direct Doppler-shift measurements are an alternative way to derive the velocity field. However, it is very difficult to obtain spectroscopic measurements with a sufficient accuracy at different angular positions to get a velocity image. So far, most observational efforts with Doppler measurements of dense planetary atmospheres in the visible domain have been dedicated to Venus and Jupiter, for two distinct reasons.

Doppler spectrometry had been envisioned for Venus since the 1970 s to understand its atmospheric circulation as it is a mostly featureless planet (Traub and Carleton, 1975, Young et al., 1979). Starting in 2007, a significant international effort was organized to support the observations of Venus by the ESA mission Venus Express (Lellouch and Witasse, 2008). The objective was to measure the atmospheric circulation using different spectral ranges, to probe different altitudes in the Venus mesosphere. Significant results on the upper mesospheric dynamics were obtained using mid-infrared heterodyne, millimeter and sub-millimeter wave spectroscopy (Sornig et al. 2008 , 2012; Clancy et al., 2008, 2012; Moullet et al., 2012). In the visible domain, observations of solar Fraunhofer lines scattered by Venus clouds have constituted most of the ground-based wind measurements (Widemann et al., 2007, 2008; Gabsi et al. 2008, Gaulme et al., 2008b; Machado et al., 2012, 2014, 2017, Gaulme et al., submitted). Regarding other planets, similar measurements were performed in the $\mathrm{mm} / \mathrm{sub}-\mathrm{mm}$ domain for Mars (Lellouch et al., 1991; Moreno et al., 2009) and Titan (Moreno et al., 2005), as well as in the infrared with the 10- $\mu$ m heterodyne observations of Titan by Kostiuk et al. (2001, 2005, 2006, 2010). It should be noticed that Doppler measurements of atmospheric dynamics on exoplanets start to be possible. Not only was it possible to measure rotation rate on several exoplanets detected by direct imaging (see for instance Snellen et al. (2014)), but wind velocities has also been reported by measuring Doppler shift of planetary spectral lines in the visible and IR domain during transit (Louden and Wheatley, 2015; Brogi et al., 2016).

The use of Doppler spectrometry for giant planets, and Jupiter in particular, was inspired by its success with helioseismology to probe its deep internal structures (e.g., Appourchaux and Grundahl, 2015). The principle relies on the detection of global oscillation modes, whose properties are a function of the internal density profile. Giant planets, being mostly fluid and convective, have seismic properties much closer to those of solar-like stars than of terrestrial planets. The basic principle relies on monitoring the position of a spectral line that probes an atmospheric level where the amplitude of acoustic modes is maximum. So far, resonant cell (Schmider et al., 1991, Cacciani et al. 2001) and Fourier Transform Spectrometry (FTS, e.g., Mosser et al., 1993, Schmider et al., 2007;, Gonçalves et al., 2016) have been considered. 
145 Two dedicated instruments were subsequently designed: the SYMPA (Seismic Interferomtric Imager for Monitoring Planetary Atmosphere) instrument was an FTS based on a Mach-Zehnder interferometer (Schmider et al., 2007) which ${ }_{205}$ has provided so far the clearest observational evidence of

150 Jupiter's oscillations (Gaulme et al. 2011). Since then, the new Doppler Spectro Imager (DSI), a descendant of SYMPA, has been developed and tested to perform both atmospheric dynamics of dense atmospheres and seismic measurements of Jupiter ${ }_{210}$ and Saturn in the framework of the two projects JOVIAL 55 and JIVE in NM.

The Doppler spectro-imager JOVIAL-JIVE is dedicated to study both internal structures and atmospheric dynamics of the giant planets of the solar system, through Doppler veloc-215 ity mapping in the visible domain. Studies of DSI were ini160 tially undertaken for Laplace, a mission project dedicated to the Jupiter system, proposed to the ESA Cosmic Vision program. Laplace then turned into JUpiter ICy moons Explorer (JUICE), which was selected as the first Cosmic-Vision L-class mission. DSI was part of the preliminary definition document of Laplace to study the formation and evolution of Jupiter's system. The payload was not selected in the end of a 5-year pro-220 cess, but in the course of the feasibility study, we developed a prototype. The design of the instrument was mainly driven by constraints arising from seismology. The project was then transformed into a ground-based multi-site network, aimed at the installation of three identical instruments on three tele-225 scopes located around the Earth, in France (Calern), the USA (Sunspot, NM), and Japan (Okayama) by the end of 2018.

Even from the ground, with limited spatial resolution, this instrument has a unique capability of providing Doppler-velocity maps. Indeed, other works dealing with measurements of 230 planetary atmospheric dynamics with visible spectroscopy met some limitations related to the sequential way of acquiring data. Regarding Venus, the techniques that have been employed so far are high-resolution échelle spectroscopy with single optical fiber feeding (Widemann et al., 2007, 2008, Gabsi et al. ${ }^{235}$ 2008, Machado et al., 2014, 2017), and long-slit spectrometry (Gaulme et al., 2008b; Machado et al., 2012, and Gaulme et al., submitted). None of these approaches provide direct imaging of velocity fields. They are therefore sensitive to image reconstruction problems and guiding errors. Beyond single-fiber ${ }^{240}$ or long-slit spectrometers, spectral imaging is possible with integral field spectrographs, which are made of different spatial channels (e.g., fiber bundles with the survey Mapping Nearby Galaxies at APO (MaNGA) within the Sloan Digital Sky Survey, described in Bundy et al., 2015). However, the disconti-245 nuities in the space domain caused by spaces in between the channels can generate discontinuities in the spectral domain. DSI is so far the only instrument able to provide instantaneous radial-velocity imaging of planets. In Gaulme et al. (2018), we carefully explore the use of line-of-sight Doppler measurements to recover the velocity field at the surface of planets of the Solar System, taking into account the many effects 250 that could affect the measurements, such as the so-called 200 Young effect, the Point Spread Function (PSF) and guiding effects, and developed the best methods to convert Doppler data into physical quantities.

In this paper, we present the first scientific results of the JOVIAL-JIVE project that were obtained with the prototype instrument at the Calern observatory, near Nice (France). We report the first measurement ever of Jupiter's average wind profile obtained with Doppler spectroscopy in the visible. The planet was observed during two runs, in 2015 and 2016 at Jupiter's oppositions, while the instrument was mounted at the foci of two telescopes: the 1.5-m MéO (Métrologie Optique) telescope in 2015 and the 1-m C2PU (Centre Pédagogique Planète Univers) telescope in 2016. Since this paper is the first report of JOVIAL-JIVE observations, we extensively describe the instrument concept, theoretical performance, and data processing method in the next two sections. Then we present the two average wind profiles of Jupiter, which we compare to simultaneous cloud-tracking data obtained by Johnson et al. (2018).

\section{How the instrument works}

\subsection{Instrumental concept}

The Doppler Spectro-Imager (DSI) is an instrument that produces radial velocity maps of extended objects. Its concept and performance are described in two SPIE papers by Soulat et al. (2012) and Gonçalves et al. (2016). The instrument is a compact Mach-Zehnder (MZ) interferometer with a fixed Optical Path Difference (OPD) which provides four interferograms, whose fringes are in phase quadrature from channel to channel. The schematic concept of the MZ is illustrated in Fig. 1. The first beam splitter (BS) separates the incoming light into two beams. The second one recombines the beams and provides two outputs with, theoretically, opposite fringe patterns. At each output, the beams are split into two polarization components by a polarizer beam splitter (polarization cube PC), which ends up producing four images. The presence of a quarter wave plate on one of the arms of the MZ generates a $\pi / 2$ phase shift between the opposite polarizations, and thus a $\pi / 4$ phase shift between each of the four outputs of the instrument. Finally, the other arm of the MZ includes a movable plate system that allows for modulating the OPD for calibration purpose. The calibration process consists of providing an instrumental response map of the four outputs, which can be obtained with the help of a uniform velocity field such as the scattered solar light in the sky (see Sect. 4). The calibration plate is eventually stabilized at its nominal position during normal observations.

The intensity of the four outputs of the instrument on the CCD detector can be described as:

$$
I_{i}(x, y)=\frac{I_{0}(x, y)}{4}\left[1+\gamma(x, y) \cos \Phi_{i}(x, y)\right],
$$

where $i$ is the index of each of the interferometer's output $(i \in[1,4]), I_{0}$ the continuum component of interferogram, $\gamma$ the fringe contrast, and $\Phi_{i}$ the phase of the fringes at each output, with $\Phi_{i}=\Phi_{0}+(i-1) \pi / 2$. The $(x, y)$ coordinates refer to the detector's coordinate and points out which terms are spatially variable. Note that the continuum term $I_{0}$ is the optical image 


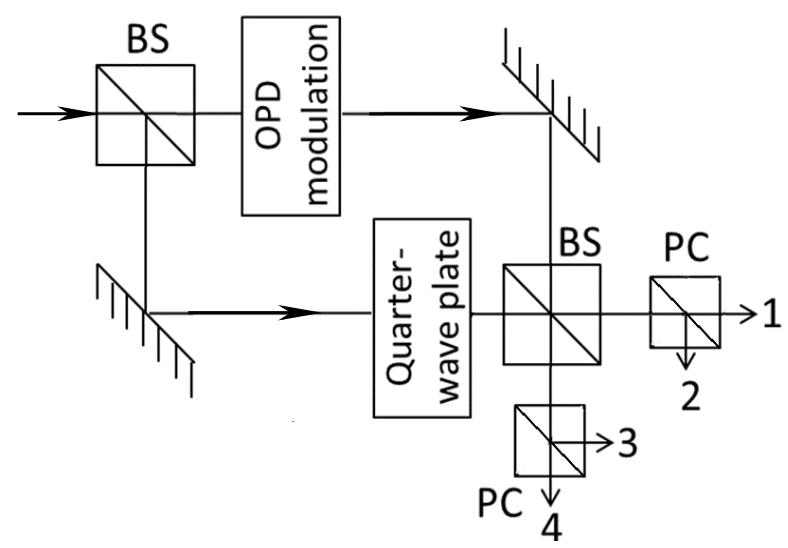

Figure 1: Conceptual design of the Mach-Zehnder interferometer. The instrument combines beam splitters (BS) and polarizing cubes (PC) to provide four ${ }_{29}$ images with interferometric fringes separated by $\pi / 2$ thanks to a quarter-wave plate. The Optical Path Difference (OPD) between the arms is fixed to $5030 \mu \mathrm{m}$ to maximize the sensitivity. The OPD can be modulated for calibration of the response of each output.

of the considered target. The phase of the interferogram is a function of the central wavenumber (inverse of wavelength) of the entrance filter $\sigma_{0}$ of the OPD $\Delta$, and of the Doppler velocity ${ }^{30}$ field $v_{\mathrm{D}}$ :

$$
\Phi_{i}(x, y)=2 \pi \sigma_{0} \Delta(x, y)\left(1+\frac{v_{D}(x, y)}{c}\right)+\phi_{i},
$$

where $\phi_{i}$ the phase shift of each output. With a perfect phase ${ }^{305}$ quadrature $\phi_{i}=(i-1) \frac{\pi}{2}$. As we discuss later, the phase quadrature is not reached in practice. In the following we usually drop the $(x, y)$ dependence to ease the reading.

We define the instrument sensitivity as the amplitude of the phase shift of an interference fringe that corresponds with a 1-m $265 \mathrm{~s}^{-1}$ radial-velocity field:

$$
S=\frac{d \Phi}{d v_{D}}=\frac{2 \pi \sigma_{0} \Delta}{c} .
$$

The standard deviation of the velocity measurements due to photon noise is expressed as:

$$
\delta v=\frac{\sqrt{2}}{\bar{\gamma}} \frac{1}{S \sqrt{N}},
$$

where $N$ is the number of photons received on the detector (Schmider et al., 2007). We here note $\bar{\gamma}$ the mean fringe contrast, which is slowly varying spatially and in between the four channels.

The above relation indicates that high-performance Doppler velocimetry must optimize the product of the sensitivity by the ${ }^{320}$ square root of the bandwidth. The optimal OPD was determined by exploring the whole solar spectrum (which Jupiter reflects back) with variable OPD and optical bandwidth. An optimum sensitivity is found for a $5190-\mu \mathrm{m}$ OPD. The optimal wavelength is found at $\lambda_{0}=519.4 \mathrm{~nm}$, where the solar spec-325 trum is rich in absorption lines that are regularly spaced ${ }^{2}$. Reg-

\footnotetext{
${ }^{2}$ The value $\approx 10^{4}$ of the ratio of the OPD to the optimal wavelength is a pure coincidence.
}

ular line spacing is a key ingredient to maximize the contrast of interferometric fringes. Maximum fringe contrast is found by setting the entrance filter's width at $1 \mathrm{~nm}$ around the central 85 wavelength $\lambda_{0}$.

Thanks to the phase quadrature of the four interferograms, it is possible to extract the phase of the interference fringes for each point of an image by the so-called ABCD method (Wyant, 1975). We then get the radial velocity at each point of the target from the phase of the interferogram, after removing from it the instrumental component that we know from the calibration procedure.

\subsection{Dealing with several layers of radial velocity fields}

Any Doppler shift of the solar spectrum induced by a motion of the reflective surface - Jupiter's upper clouds - results in a shift of the phase of the interferogram. According to Eq. 2 the phase shift $\delta \Phi(x, y)$ is expressed as:

$$
\delta \Phi(x, y)=S(x, y) v_{\mathrm{D}}(x, y) .
$$

We stress that the sensitivity $S$ is variable across the field of view because the OPD is a function of the varying incidence angle on the $\mathrm{MZ}$ prisms.

The Doppler shift of solar lines that are reflected on Jupiter is the combination of the relative motion of Jupiter with respect to the $\operatorname{Sun}\left(v_{\mathrm{J} / \mathrm{S}}\right)$, to the Earth barycenter $\left(v_{\mathrm{E} / \mathrm{J}}\right)$, and to the observer who is located on the rotating Earth $\left(v_{\mathrm{E}, \mathrm{rot}}\right)$. Therefore, a given point at the surface of Jupiter feels the incident light coming from the Sun with a relative radial velocity:

$$
v_{\mathrm{i}}=v_{\mathrm{J} / \mathrm{S}}+\tilde{v}_{\mathrm{J}, \text { rot }}+\tilde{v}_{\mathrm{wind}},
$$

where we split the radial velocity field associated with Jupiter's rotation into a mean solid body $\tilde{v}_{\mathrm{J}, \text { rot }}$ and a wind $\tilde{v}_{\text {wind }}$ component which is $(x, y)$-dependent. The same given point reflects the light towards the observer with a relative radial velocity:

$$
v_{\mathrm{e}}=v_{\mathrm{E} / \mathrm{J}}+v_{\mathrm{E}, \text { rot }}+\tilde{v}_{\mathrm{J}, \text { rot }}+\tilde{v}_{\text {wind }} .
$$

Thus, the total radial velocity map is the sum of the relative projected motions:

$$
v_{\mathrm{D}}=v_{\mathrm{E} / \mathrm{J}}+v_{\mathrm{E}, \text { rot }}+v_{\mathrm{J} / \mathrm{S}}+2\left(\tilde{v}_{\mathrm{J}, \text { rot }}+\tilde{v}_{\text {wind }}\right) .
$$

However, the above expression is only true when the source, target and the observer are aligned, i.e., when the planet is seen at opposition, and when we work with reflected solar lines. In this precise case, the instrument sensitivity to proper motions of the surface of the considered planet is doubled (factor of 2 in Eq. 8).

In the general case, for any planetary target $T$ seen at whatever phase angle $\varphi$-Sun-target-observer angle - the coefficient that magnifies (or not) the amplitude of motions at the surface of the planet is $(1+\cos \varphi)$ when we work with reflected solar lines. Note that there is no magnification at all in the case of intrinsic planetary lines, as molecular methane absorption on Jupiter or carbon dioxide on Venus, which is not the case of 
${ }_{330}$ JOVIAL-JIVE. Therefore, in the general case of a planet observed form Earth, we write:

$$
v_{\mathrm{D}}=v_{\mathrm{E} / \mathrm{T}}+v_{\mathrm{E}, \mathrm{rot}}+\zeta v_{\mathrm{T} / \mathrm{S}}+\kappa\left(\tilde{v}_{\mathrm{T}, \mathrm{rot}}+\tilde{v}_{\mathrm{wind}}\right),
$$

where

$$
\begin{aligned}
& \zeta=1 \text { and } \kappa=(1+\cos \varphi) \text { for reflected solar lines } \\
& \zeta=0 \text { and } \kappa=1 \text { for planetary lines }
\end{aligned}
$$

From Eq. 2, the phase map is the sum of two contributions: an instrumental term $\Phi_{\text {instr }} \equiv 2 \pi \sigma_{0} \Delta$, and a Doppler velocity term $\Phi_{\mathrm{D}} \equiv 2 \pi \sigma_{0} \Delta v_{\mathrm{D}} / c$. In this paper, the whole purpose of the data processing chain is to first remove the instrumental contribution $\Phi_{\text {instr }}$ from the interferograms, then disentangle the several components of the velocity field to extract the $\tilde{v}_{\text {wind }}$ $\Phi_{\mathrm{D}}$. In the following, we consider only the case of JOVIAL-JIVE, for which reflected solar lines are used, therefore $\zeta$ is equal to 1 and $\kappa$ is $1+\cos \varphi$.

\subsection{From four interferograms to a velocity map}

If we now consider the two pairs of interferograms with opposite phases, it is possible to cancel the continuous component $I_{0}$ of the interferograms by combining them. We get pure and ${ }^{375}$ normalized interferograms $U$ and $V$ by computing:

$$
U=\frac{I_{1}-I_{3}}{I_{1}+I_{3}} \quad V=\frac{I_{2}-I_{4}}{I_{2}+I_{4}},
$$

where $I_{i}$ with $i \in[1,4]$ are the four images, and where odd even subscripts correspond to equal polarizations and opposite phases. If the phase quadrature between images is ${ }^{380}$ respected, then we have

$$
U \propto \cos \Phi \quad V \propto \sin \Phi
$$

Since the Doppler signal is stored in the phase of the interferograms, we need to extract the phase from $U$ and $V$. This ${ }^{385}$ can be achieved by first computing the complex interferogram $Z$, defined as:

$$
Z=U+i V \propto e^{i \Phi} .
$$

The argument of $Z$ is the phase map of the interferogram. However, to extract the Doppler contribution from the phase of $Z$, we need to multiply $Z$ with the complex conjugate of the instrumental component $Z_{\text {instr }}$ obtained from the calibration process, and then compute its argument. The Doppler velocity field $v_{\mathrm{D}}$ is embedded in the phase of the complex interferogram $Z_{\mathrm{D}}$ :

$$
Z_{\mathrm{D}}=Z Z_{\text {instr }}^{*} \propto \exp \left(i S v_{D}\right)
$$

To eventually focus on the differential rotation of Jupiter i.e., the winds - we need to repeat the process by multiplying $Z_{\mathrm{D}}$ with the complex conjugate map of the solid-body radial-400 velocity field of Jupiter $Z_{\mathrm{J}, \text { rot }}=\exp \left(i S \kappa \tilde{v}_{\mathrm{J}, \text { rot }}\right)$. In practice, the solid-body term $Z_{\mathrm{J}, \text { rot }}$ is obtained with a theoretical model of the planet, which includes its orientation and oblateness (see Appendix A. The radial-velocity map of the winds is the spatiallyvariable part of argument of the complex quantity $Z_{\text {wind }}$ :

$$
Z_{\text {wind }}=Z_{\mathrm{D}} Z_{\mathrm{J}, \text { rot }}^{*} \propto \exp \left[i S\left(v_{\text {unif }}+\kappa \tilde{v}_{\text {wind }}\right)\right]
$$
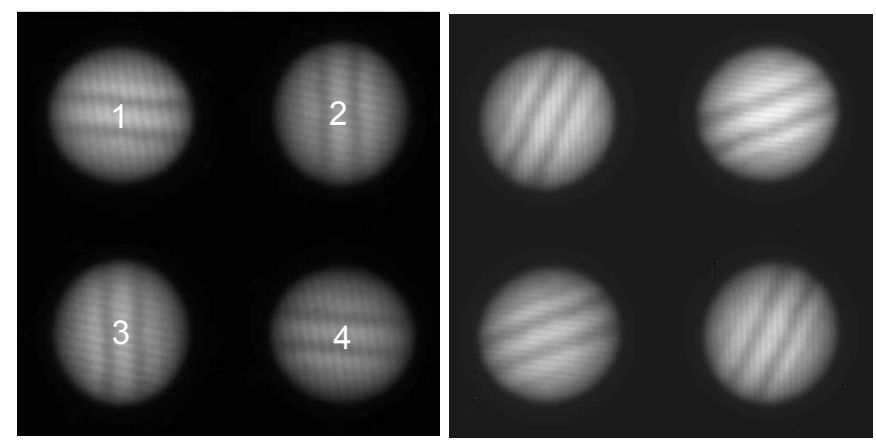

Figure 2: Raw CCD images of acquisitions on Jupiter during the 2015 (left) and 2016 (right) observation runs. Each acquisition contains four sub-images of Jupiter corresponding to the four outputs of the instrument. Each row displays two images corresponding to the two different outputs of the Mach-Zehnder, each line contains images of the same polarization. The orientation of the outputs is due to the optical configuration of the instrument and has no special meaning. The orientation of Jupiter within the field changes with time due to the field rotation at the coude focus. The interferometric fringes are visible on each image. The fringe period is shorter in 2016, as explained in Table 1 The contrast is about $3 \%$. The phase of the fringes is separated by almost $\pi / 2$ (quadrature) between images.

where $S$ is the instrument velocity sensitivity defined by Eq. 3 . $\tilde{v}_{\text {wind }}$ the velocity of the Jovian winds which is $(x, y)$-dependent, and $v_{\text {unif }}=v_{\mathrm{E} / \mathrm{J}}+v_{\mathrm{E}, \text { rot }}+v_{\mathrm{J} / \mathrm{S}}$ which is the uniform radial-velocity component.

\section{Actual performance and data processing}

\subsection{Issues caused by geometrical distortions}

The raw data consists of four images of Jupiter $\left(I_{1}, I_{2}, I_{3}, I_{4}\right)$ displayed on a single CCD square chip (Fig. 2). The preprocessing steps consist of cleaning, cutting out and adjusting each image to create the normalized interferograms $(U, V)$. We first work with the whole frame - the four images together - to subtract the dark current of the camera to eliminate the thermal noise. Then, we divide each frame by a flat-field image to eliminate the inhomogeneous response of the CCD pixels. Note that obtaining a flat field for a spectral device is not an easy task. Generally, for astronomical observations, flat fields are obtained by enlightening an isotropic white surface or by taking data on the cloud-clear twilight sky. For an interferometer, such an approach is not possible because either the lamp used to enlighten a white surface or the sky may display spectral lines that could cause low-contrasted interference fringes, which would bias the flat-field image. We managed to get proper flat-field calibration data by modulating the OPD with the piezo-electric system during exposures taken on the blue sky. During flat-field exposures, the OPD is continuously modulated by steps of one wavelength to blur the fringes and cancel them out. We remove the hot pixels and cosmic rays replacing them with the average values of their neighboring pixels. The pre-processed CCD image of $1024 \times 1024$ pixels is then cut into four equal size images of $512 \times 512$ pixels.

The computation of the Jupiter phase map requires that the four images overlap within an accuracy better than 1/10 pixels. This constraint is driven by the goal of keeping the error 
introduced by overlapping the images below a given level. The goal of a tenth of a pixel was determined from simulations of the instrument, when we aim at keeping the noise introduced by image recombination lower than the photon noise. Repositioning the four images so finely requires an accurate calibration of the optical device to know the parameters of the image trans-460 formations: translation, rotation, scale and optical distortion.

The optical distortion has a component that is common to all four images, and a differential one that is specific to each channel. To limit the impact of interpolation on the phase of the interferogram, we first measure all terms related to optical ${ }^{465}$ distortion and apply a correction once, at the end of the data Also, we do not aim at rectifying the images in an absolute manner: instead, we aim at getting the four images of each exposure to be superimposable and the radialvelocity maps to be superimposable as function of time. There-470 fore, we select a reference image based on SNR criteria, and we image.

We perform the affine transformations (translation, rotation and scale) with a method based on Fourier transform, which gives more accurate results than other interpolation methods. 475

430 This method is based on the shifting and scaling properties of the Fourier transform:

$$
\begin{aligned}
f\left(x-x_{0}, y-y_{0}\right) & \longleftrightarrow F(u, v) e^{-2 \pi\left(u x_{0}+v y_{0}\right)} \\
f(a x, b y) & \longleftrightarrow \frac{1}{|a b|} F\left(\frac{u}{a}, \frac{v}{b}\right)
\end{aligned}
$$

To perform the rotation operation, we make use of the 3-shear transform method (Owen and Makedon, 1996). This approach has the advantage of better preserving the spatial spectral in- ${ }_{485}$ mation than other numerical methods. We tested different methods by first applying a transform on an image and then the inverse transform. The result is compared to the initial image. The differences found with this method are much lower than with any other interpolation methods. Note that computing the ${ }_{490}$ 40 transformations in the Fourier domain requires smoothing the image edges to avoid high spatial frequency noise caused by abrupt edges.

\subsection{Measuring the optical distortion}

The process used to measure the distortions is similar to what was developed for processing the SYMPA data (see Gaulme et al. 2008b, for a comprehensive explanation). In brief, we determine the transformation parameters from a calibration grid,500 which consists of a slice of glass with laser-incised dots, that is placed at the focal plane of the instrument. Eventually, the

450 knowledge of the set of distorted coordinates is used to build the new rectified image by interpolating the image on the regular detector coordinates. The number of dots is about $50 \times 50_{505}$ across the FOV. In practice, over a 1200 points are used to rectify the images. To estimate the distortion of the FOV, it is nec-

455 essary to precisely know the position of the dots in each image. The position of the dots is obtained by fitting each one with an ellipse. An empirical expression of the photometric profile of $f_{510}$ each dot upon the detector is:

$$
P(x, y)=\pi / 2-\arctan ^{4}\left(\left(\frac{x-x_{0}}{\sigma_{x}}\right)^{2}+\left(\frac{y-y_{0}}{\sigma_{y}}\right)^{2}\right)
$$

This process permits to determine the dot positions with an accuracy better than $1 / 50$ pixel. Once the coordinates of each dot for each image are measured, we can determine the transformations to be applied to the coordinate frames of each image.

The relative distortion map is fitted with a two-dimensional third-degree polynomial. The amplitude of relative distortion is of the order of one pixel, with a typical standard deviation of about 0.1 pixel. The interpolation is performed with a cubic spline algorithm. We finally estimate the efficiency of the rectification by applying the transformation to the set of four calibration grid images. It appears that residual differences between the coordinates of an image with respect to the reference one are less than 0.04 pixel with a standard deviation of about 0.007 pixel.

\subsection{Measuring the instrumental response}

According to Sect. 3.3 , it is necessary to measure the complex expression of the instrumental fringes $Z_{\text {instr }}$ to get the complex interferogram $Z_{\mathrm{D}}$ that corresponds with the Doppler signal (see Eq. 15. To do so, we would need a uniformly illuminated field with solar light and a uniform velocity field. For this, we point the telescope at the blue sky (or white clouds) during the day. We then acquire four successive images with a shift of the OPD in steps of $\lambda / 4$ thanks to the piezoelectric plate of the MZ. The four images are then separated by $\pi / 2$ in phase. We can extract a complete mathematical description of the instrumental fringes in the field (fringes contrast and phase, relative photometric response) for each channel with the ABCD method (Fig. 3)

This calibration is realized by acquiring data on the day sky, ideally when it is covered by uniform white clouds. Indeed, a uniform cloud layer makes the sky brighter and less polarized than the blue sky, providing a better calibration. Acquisitions are required to last over an hour to get a sufficient SNR. In principle, one calibration map would be sufficient to analyze a whole observing run. However, as subtle telescope/instrument configurations could happen, we opt for having one calibration per day, weather permitting.

Figure 4 displays the relative phase maps of each output. It appears that the condition of having a phase quadrature between each output is not perfectly met: we observe a phase difference of about $101.4^{\circ}$ between $\Phi_{2}$ and $\Phi_{1}$ and about $104.6^{\circ}$ between $\Phi_{4}$ and $\Phi_{3}$, instead of $90^{\circ}$. The origin of this departure is likely the result of imperfections of the optical device, in particular the beam splitters and the quarter-wave plate. Such a departure from phase quadrature was also observed with the SYMPA instrument and methods to cope with it were proposed by Gaulme et al. (2008a). We discuss the implication of departure from phase quadrature on data processing and on the quality of radial-velocity measurements in Appendix B.

After correction of the departure from the phase quadrature, we get rid of the instrumental contribution to the interferograms 

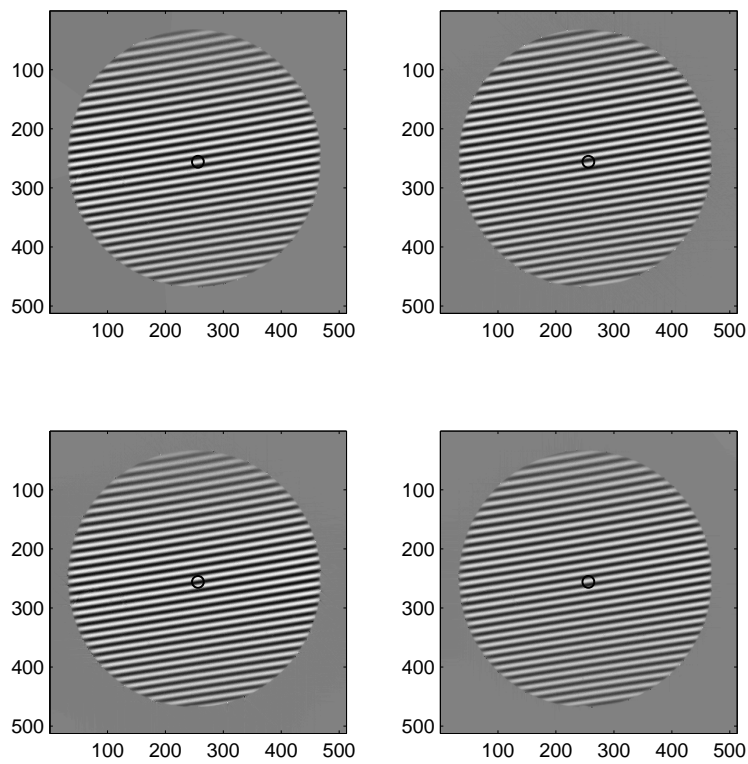

Figure 3: Instrumental fringes (real part of $Z_{\text {instr }, i}$ ) for the four instrumental outputs obtained from the calibration process.

in the same way as for perfect quadrature (see Eq. 15):

$$
Z_{\mathrm{D}}^{\prime}=Z^{\prime} Z_{\text {instr }}^{\prime *}
$$

where the calibration interferogram $Z_{\text {instr }}^{\prime}$ is created in the same $e_{535}$ way as $Z$ '. With respect to the perfect quadrature case, the noise level on the phase is increased by a factor $1 / \cos \epsilon$.

\subsection{Correction of non-uniform relative flux}

An unexpected problem that appeared by processing actual ${ }^{540}$ data is that the pairs of images $I_{1}, I_{3}$ and $I_{2}, I_{4}$ do not display a uniform relative flux, despite flat-field correction. This nonuniformity generates spurious fringes. The origin of this problem is unclear, but is probably related to the polarization of the instrument coupled with the rotation of the FOV throughout the ${ }^{545}$ night, caused by the fact that the instrument was mounted at telescopes' coudé foci. To solve this problem, we apply a photometric correction to each acquisition. The correction consists of two steps: we first filter the spurious fringes out of each image $I_{i}, i \in[1,4]$ in their 2-D Fourier transform, and then ${ }^{550}$ convolve them with a Gaussian function to smooth the map of the photometric correction. Then, we build the photometrically

$$
I_{3}^{\prime}=\frac{\tilde{I}_{1}}{\tilde{I}_{3}} I_{3} \quad \text { and } \quad I_{4}^{\prime}=\frac{\tilde{I}_{2}}{\tilde{I}_{4}} I_{4}
$$

where $\tilde{I}_{i}$ are the filtered images. This photometric correction does not exceed a few percent of the maximum intensity, and it eliminates all of the undesired fringes.
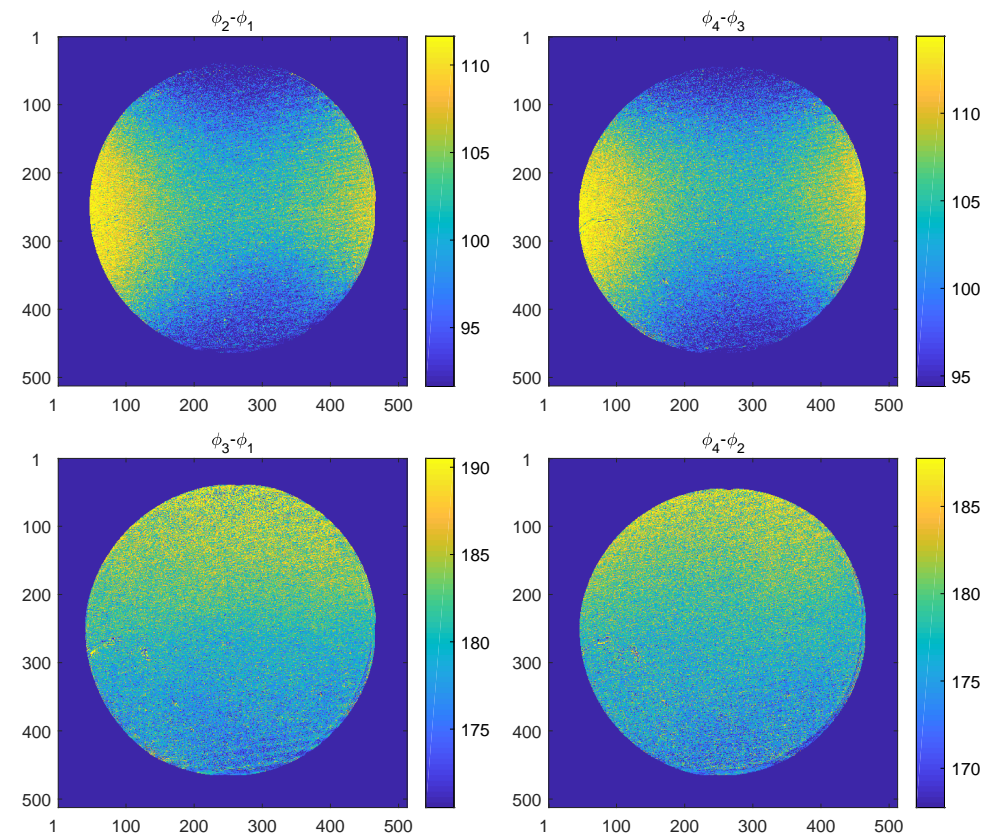

Figure 4: Instrumental phase difference between outputs of the MZ in degrees. The two images on top correspond to phase differences between outputs with opposite polarization (2-1 and 4-3) and the two images below to the phase difference for the output with same polarization (3-1 and 4-2).

\subsection{Testing the data processing with simulations}

To test the complete data processing pipeline, we produced simulations of the whole CCD frame that include the quadruplet of Jupiter interferograms. The interferograms take into account the actual figure of Jupiter in the visible for the photometric part, and the solid-body rotation plus the mean zonal wind profile for the radial velocity part.

The continuum component $I_{0}(x, y)$ was obtained by "painting" a model of Jupiter, i.e., an oblate ellipsoid oriented as Jupiter was during our observations, with an actual planisphere of the planet in the visible (see Appendix A. The visible map of Jupiter was made and posted by Flavio Fortunato (www.astrobin.com/161663/b) based on lucky imaging observations at the same epoch as our observations. The maps were generated with a resolution of $0.1 \mathrm{deg} / \mathrm{pix}$, between $-79.8^{\circ}$ and $+79.8^{\circ}$ planetographic latitudes.

For the wind circulation model, we used the planetocentric zonal wind profiles obtained by Johnson et al. (2018) from cloud-tracking measurements obtained with the Hubble space telescope as part of the OPAL program in 2015 and 2016 (Simon et al., 2015). More precisely, the HST data were acquired with the Wide Field Camera (WFC3/UVIS) on Jan. 19, 2015 between 02:00 and 23:40 UT (this is about a month earlier than the DSI observations) in the F502N green filter. The maps used for the 2016 extraction were composed of Jupiter data acquired with WFC3/UVIS between Feb. 9, 2016 09:35 and Feb 10, 

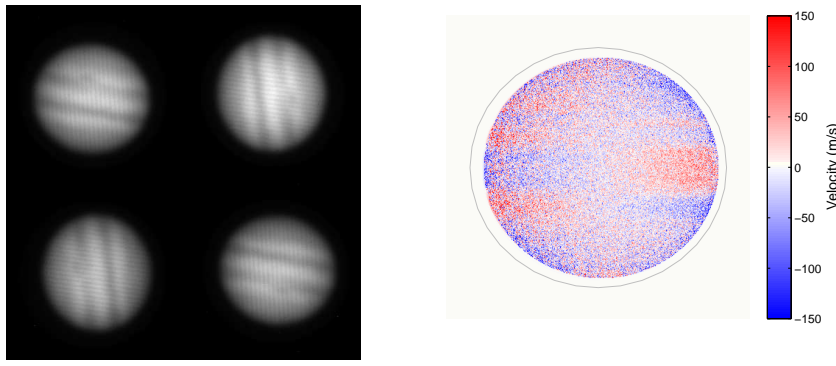

Figure 5: Left: simulated CCD image. Right: Example of simulation results ${ }^{595}$ for cumulative residual velocity for one night with parameters corresponding to the observation of $02 / 18 / 2015$. The zonal winds introduced in the noisy model appear clearly.

Table 1: Mach-Zehnder settings for the two observation cam-600 paigns.

\begin{tabular}{cccccc}
\hline Year & $\begin{array}{c}\text { OPD } \\
{[\mu \mathrm{m}]}\end{array}$ & $\begin{array}{c}\text { Fringe } \\
\text { contrast }\end{array}$ & $\begin{array}{c}\text { Sensitivity } S \\
{\left[\mathrm{rad} \mathrm{km}^{-1} \mathrm{~s}^{-1}\right]}\end{array}$ & $\begin{array}{c}\text { Fringe period } \\
\text { pixel \# }\end{array}$ & $\begin{array}{c}\text { Pixel FOV } \\
{[\operatorname{arcsec}]}\end{array}$ \\
\hline 2015 & 5140 & $3.5 \%$ & 0.4 & 20 & 0.128 \\
2016 & 5190 & $4.3 \%$ & 0.4 & 13 & $0.13{ }_{665}$ \\
\hline
\end{tabular}

2016 05:12 UT (also about a month earlier than the DSI observations) in the F502N green filter. Note that we transform ${ }^{610}$ the planetographic $\left(\lambda_{\mathrm{g}}\right)$ latitudes into planetocentric $\left(\lambda_{\mathrm{c}}\right)$ by using the relation:

$$
\tan \left(\lambda_{\mathrm{c}}\right)=\left(\frac{R_{p}}{R_{e q}}\right)^{2} \tan \left(\lambda_{\mathrm{g}}\right),
$$

where $R_{p} / R_{e q}$ is the ratio of the polar radius to the equatorial radius (Beebe, 1994).

From the simulated model, we calculate four sub-images with interferometric fringes, and apply the geometrical transthe reproduce the distortions. The simulation also includes the photon noise, flux variations, field rotation through the night, degradation of the image by the instrumen-625 tal Point Spread Function (PSF) and the atmospheric turbulence for a typical observation night. It has to be noticed that the PSF troduces a bias in the estimation of the velocity, as shown in the companion paper Gaulme et al. (2018) (see Appendix B). We take into account 630 this effect in our simulated data. The simulated time series is then processed by the pipeline in the exact same way as science mages. Figure 5 displays an example of simulated data (left) and the mean radial velocity $\tilde{v}_{\text {wind }}$ resulting from a night of simulated data (right). No specific bias are visible on the mean ${ }_{635}$ velocity map, whereas we easily distinguish the signal caused by zonal winds. The simulations thus validate the procedure of data reduction within the limits of the assumptions taken into account. These simulations are eventually used to compare our results of the wind profile in Sect.5

\section{Observations of Jupiter in 2015 and 2016}

\subsection{Observing conditions}

Two observational campaigns were organized in 2015 and 2016 with the prototype of the MZ near the opposition of Jupiter. The settings were slightly modified between both campaigns (Table 1). The optical settings were changed in the laboratory after we updated the mechanical assembly of the $\mathrm{MZ}$ device. Also the fringe intervals were reduced to allows a correct sampling of the fringes upon the detector, and at the same time as tight as possible to keep a good-enough spatial resolution on the radial-velocity maps.

Two observation campaigns (Tables 2 and 3 were conducted at the Calern Observatory (France), the first in February 2015 with 4 useful nights ( $24 \%$ duty cycle), and the second in March 2016 with 14 exploitable nights (16.4\% duty cycle). Two different telescopes were used: the 1.5-m MéO azimuthal telescope in 2015 and the 1-m C2PU-East equatorial telescope in 2016. In both cases the DSI instrument was installed on a fixed table at the coudé focus of the telescope. Such a configuration causes the image of Jupiter to rotate in the field of view of the instrument.

The exposure time of individual images is driven by the need of sampling fast-enough the expected oscillations of Jupiter, and having a sufficient signal-to-noise ratio (SNR) of the phase at each point. Indeed, the noise on the phase must be well below $2 \pi$ for each pixel of a single image, otherwise we lose track of the absolute phase. These requirements led us to choose a sample rate of 30 s and an exposure time of 28.7 s, by taking into account the detector's read-out time. For both campaigns, the optical interface of the telescope included a fast-steering mirror to compensate for guiding errors and atmospheric seeing. Thanks to the fast-steering mirror, the position of Jupiter in the field could be stabilized to within 0.1 arcsec. In addition, during the first observing run, we observed a slow drift of the pupil that was related to the rotation of telescope. This causes the interferogram phase to slowly vary due to optical inhomogeneities in the MZ interferometer. For the second campaign, we implemented a correction of the pupil position by placing a slow steering mirror at the first focus plane at the entrance of the instrument. This allowed a stabilization of the pupil to better than $1 / 200$ of its diameter.

The optical output of the DSI is arranged to form four images of Jupiter on a FLI4710 CCD camera having 1024x1024 pixels. The field in each image is about 60 arcsec with a scale factor of 7.8 pixel $\operatorname{arcsec}^{-1}$ for 2015 and 7.6 pixel $\operatorname{arcsec}^{-1}$ for 2016. The maximum image rotation overnight was $112^{\circ}$ in 2015 and $122^{\circ}$ in 2016 . The total transmission (telescope and instrument in 1-nm band) was estimated to be $7 \%$ in 2015 and $15 \%$ in 2016. The difference of total transmission between the 2015 and 2016 runs, which is related to the number of optical devices and optical quality, was enough to compensate for the loss of collecting area between the two campaigns. In the end, the total effective flux was practically the same in both campaigns. 
Table 2: Observing conditions from the February 2015 and March 2016 campaigns conducted at the Calern Observatory.

\begin{tabular}{|c|c|c|c|c|c|c|}
\hline $\begin{array}{c}\text { Starting date (UT) } \\
\text { m-d-y, h:m:s }\end{array}$ & $\begin{array}{c}\text { Ending date (UT) } \\
\text { m-d-y, h:m:s }\end{array}$ & $\begin{array}{c}\text { \# of } \\
\text { acquisitions }\end{array}$ & \# selected & $\begin{array}{l}\text { Duration } \\
\text { h:m:s }\end{array}$ & $\begin{array}{c}\text { Mean Intensity } \\
10^{9} \text { photons image }^{-1}\end{array}$ & $\begin{array}{c}\text { Mean FWHM } \\
\text { arcsec }\end{array}$ \\
\hline $02-17-15,18: 33: 40$ & $02-18-15,04: 56: 00$ & 898 & 873 & $06: 57: 35$ & 0.87 & 3.6 \\
\hline $02-18-15,17: 13: 30$ & $02-19-15,05: 00: 00$ & 1298 & 1249 & $09: 57: 26$ & 1.05 & 3.9 \\
\hline $02-19-15,17: 39: 30$ & $02-20-15,04: 41: 00$ & 1058 & 899 & 07:10:01 & 1.14 & 4.2 \\
\hline $02-22-15,19: 31: 30$ & $02-23-15,04: 44: 00$ & 1086 & 1085 & $08: 38: 59$ & 0.95 & 3.8 \\
\hline $03-03-16,22: 58: 30$ & $03-04-16,04: 29: 00$ & 305 & 0 & 00:00:00 & - & - \\
\hline $03-05-16,21: 01: 30$ & 03-06-16, 04:18:30 & 872 & 819 & $06: 31: 45$ & 1.24 & 3.6 \\
\hline $03-06-16,20: 37: 30$ & 03-07-16, 04:16:30 & 719 & 619 & 04:56:05 & 1.25 & 2.4 \\
\hline $03-07-16,19: 55: 00$ & $03-08-16,04: 12: 30$ & 949 & 898 & 07:09:32 & 1.27 & 3.6 \\
\hline $03-08-16,19: 56: 00$ & 03-09-16 04:08:30 & 956 & 943 & 07:31:04 & 1.32 & 3.5 \\
\hline $03-09-16,22: 05: 00$ & $03-10-16,02: 10: 30$ & 470 & 337 & 02:41:11 & 0.65 & 3.0 \\
\hline $03-10-16,19: 18: 30$ & $03-11-16,04: 00: 00$ & 987 & 841 & $06: 42: 16$ & 1.17 & 2.8 \\
\hline $03-11-16,20: 20: 30$ & $03-12-16,02: 00: 30$ & 244 & 166 & $01: 19: 24$ & 0.64 & 2.5 \\
\hline $03-12-16,19: 52: 00$ & $03-13-16,03: 51: 30$ & 986 & 968 & 07:43:01 & 1.11 & 2.5 \\
\hline $03-14-16,20: 02: 00$ & 03-15-16, 03:51:30 & 850 & 822 & $06: 33: 11$ & 1.10 & 2.3 \\
\hline $03-18-16,19: 03: 30$ & $03-19-16,03: 26: 30$ & 988 & 982 & $07: 49: 43$ & 1.24 & 2.8 \\
\hline $03-19-16,21: 00: 00$ & 03-20-16, 02:13:00 & 626 & 594 & 04:44:07 & 1.17 & 2.8 \\
\hline $03-20-16,20: 47: 00$ & $03-21-16,22: 56: 00$ & 248 & 230 & 01:50:01 & 0.70 & 2.9 \\
\hline $03-21-16,20: 19: 00$ & $03-22-16,03: 13: 30$ & 633 & 607 & 04:50:20 & 1.11 & 3.0 \\
\hline $03-22-16,19: 05: 30$ & 03-23-16, 00:26:00 & 17 & 0 & 00:00:00 & - & - \\
\hline $03-23-16,18: 20: 30$ & $03-24-16,03: 05: 00$ & 971 & 910 & $07: 15: 17$ & 0.85 & 3.4 \\
\hline
\end{tabular}

Table 3: Astronomical and physical configurations of Jupiter during the 2015 and 2016 campaigns.

\begin{tabular}{lrc} 
& 2015 & 2016 \\
\hline RA [h] & 09.23 to 9.20 & 11.32 to 11.18 \\
Dec [deg] & 16.98 to 17.28 & 05.93 to 06.85 \\
VMag & -2.55 to -2.53 & -2.49 to -2.46 \\
Equatorial diameter [arcsec] & 44.8 to 44.6 & 44.1 to 43.8 \\
Max. altitude [deg.] & 63.15 to 63.30 & 52.10 to 53.00 \\
Phase [deg.] & 2.420 to 3.420 & 0.340 to 3.200670 \\
Velocity J/E [km.s ${ }^{-1}$ ] & 6.3 to 9.0 & -1.6 to 8.17 \\
Amplitude of Earth rot. [km. ${ }^{-1}$ ] & 0.32 & 0.33 \\
Time of altitude $>20$ deg & $10 \mathrm{~h} 20$ & $9 \mathrm{~h} 00$ \\
Opposition date & $02-06-2015$ & $03-08-2016$ \\
\hline SEP LAT [deg.] & -0.15 to -0.13 & -1.88 to -1.80 \\
SSP LAT [deg.] & -0.06 to -0.08 & -1.59 to 1.65 \\
NP [deg.] & 19.5 to 19.3 & 25.3 to 25.10 \\
\hline
\end{tabular}

\subsection{Phase maps and noise level}

We processed the complete times series taken during the 2015 and 2016 campaigns by following the protocol described in the previous sections. In Fig. 6, we display an example of raw data on the top left, real part of the phase as obtained by Eq. (14) (top right), then corrected from the instrumental component as in Eq. (20) and from residual fringes caused by flat-field imperfections filtered out (see 4.4) at bottom left, and finally the residual velocity field with the component of solid body rotation removed (bottom right). This protocol is applied to each acquisition. All the phase maps are rectified from optical distortions, centered and corrected from ${ }^{690}$ field rotation, so that they can be superimposed.

Once we have radial-velocity maps, a criterion to distinguish

655 "good" from "bad" data is to estimate the noise level for each pixel on each map. We can derive the variance of the phase measurements from the normalized interferograms $U$ and $V$ (see Eq. 4 and Appendix C). It is a function of the received 695 number of photons and the fringe contrast (Schmider et al. 660 2007):

$$
\langle\delta \phi\rangle^{2}=\frac{2}{N \gamma^{2}},
$$

where $N$ is the total number of photons per pixel and $\gamma$ is the 700 fringe contrast. This assumes the same flux for each image and the same phase quadrature for the four outputs of the instrument. We derive the theoretical standard deviation expected for each point on a single image from the actual number of photons for each image. In average, the theoretical noise level based on the actual fringe contrast and photon noise during the 2015 and 2016 campaigns are:// for 2015: $\delta_{\phi}=0.38 \mathrm{rad} . p x^{-1} \equiv$ 928m. $\mathrm{s}^{-1} / /$ for 2016: $\delta_{\phi}=0.32 \mathrm{rad} . \mathrm{px} x^{-1} \equiv 780 \mathrm{~m} . \mathrm{s}^{-1} / /$ for each pixel of individual images.

In 2015, the actual noise over the Jovian disk calculated as the standard deviation between images was estimated to be $1.36 \mathrm{kms}^{-1}$ in average for each image and each pixel (on 90\% of the disk). This is about $40 \%$ larger than the theoretical photon noise based on the flux measured on the detector. In 2016, we find a much better noise level of $892 \mathrm{~ms}$ ${ }^{-1}$ in average, compared to a theoretical level of $780 \mathrm{~ms}^{-1}$ of the corresponding night, again on $90 \%$ of the Jovian disk. The number of photons was comparable between 2015 and 2016, despite the smaller telescope diameter, because the transmission of the coudé train is better. Moreover, the contrast of the fringes in $\mathbf{2 0 1 6}$ was improved and the stability was better thanks to the pupil stabilization (see below), so the noise level is close to the theoretical one. As the quality of the data is better in 2016, it was possible to keep almost $99 \%$ of the Jovian disk for analysis. Given the size of Jupiter, this conducts to a dispersion of global velocity measurements obtained every 15 minutes of about $15 \mathrm{~ms}^{-1}$ in 2015 and $9 \mathrm{~ms}^{-1}$.

\subsection{Temporal and spatial evolution of velocity maps}

Assessing the long-term stability of the instrument involves monitoring the mean value of the phase of the complex interferogram $Z_{\mathrm{D}}^{\prime}$, computed on the whole disk of Jupiter (Fig. 7). This time series shows the rotation of the Earth as well as the relative velocity between Jupiter and the Earth. We observe some fluctuations around theoretical expectations, which are caused by uncertainties on the exact position of Jupiter's center on the reference image. In addition, in 2015 , the position of the pupil changed during the night due to the telescope optical configu- 

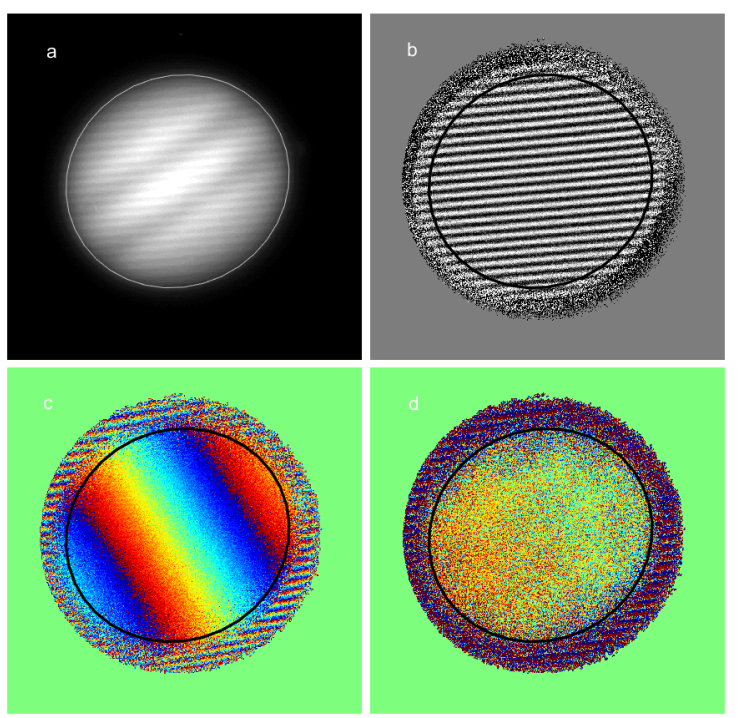

Figure 6: Top left: example of a Jupiter image obtained on March 3rd, 2016 at $\mathrm{C} 2 \mathrm{PU}$, where the rotation axis is tilted by about $45^{\circ}$ on the detector. Top right: real part of the interferogram $Z^{\prime}$ as described in Eq. 20 Bottom left: phase map of $Z_{\mathrm{D}}^{\prime}$ after subtracting the instrumental fringes from the complex interferogram $Z_{\text {instr }}^{\prime}$. It shows the rotation of Jupiter, which produces a linearly increasing phase along the equator. The color scale corresponds to $[0: 2 \pi]$. (phase jumps correspond with the phase getting larger than $2 \pi$ ). Bottom right: residual phase map, i.e., the phase of the complex interferogram $Z_{\text {wind }}^{\prime}$. The color scale is amplified by a factor of 10 , in order to show the residuals. On ${ }^{730}$ each figure, the edge of Jupiter is displayed as a plain line ellipse. A phase can be measured outside the Jupiter, because of the PSF effect.

ration, which results in variations of the phase of the interfero- ${ }_{735}$ metric fringes, as indicated in Sect. 5.1.

In 2016, the pupil was stabilized into the MZ to avoid the phase variations caused by the pupil drift throughout the night. The mean temporal phase variations are less noisy than the previous year. However, some offsets remain, which likely result ${ }_{74}$ from changes in the instrumental set-up from one day to another. First, the piezoelectric system was off during the first four nights, then switched on for calibration during two consecutive nights. This resets its position and may introduce an OPD offset. Second, long-term temperature variations may have af- 745 fected the phase when switching on the piezoelectric plate as it produces heat, even though the MZ interferometer is thermally controlled in its vacuum tank. Also, a general power flaw can generate phase discontinuities, as about 24 hours are required for the instrument to reach a thermal equilibrium. The ${ }_{750}$ first four observation nights of 2016 show no strong variations. The absolute stability for these four nights is of the order of 200 $\mathrm{m} \mathrm{s}^{-1}$, which is close to the level measured in laboratory tests (Gonçalves et al. 2016).

After removing the mean phase of each image, we remove ${ }_{755}$ from each image the instrumental phase and the solid rotation model. In the following, we call residual velocity maps the maps that are both corrected from instrumental and solid body rotation, i.e., extracted from the phase of $Z_{\text {wind }}$. The residual velocity map is also corrected from the bias arising from the ${ }_{760}$

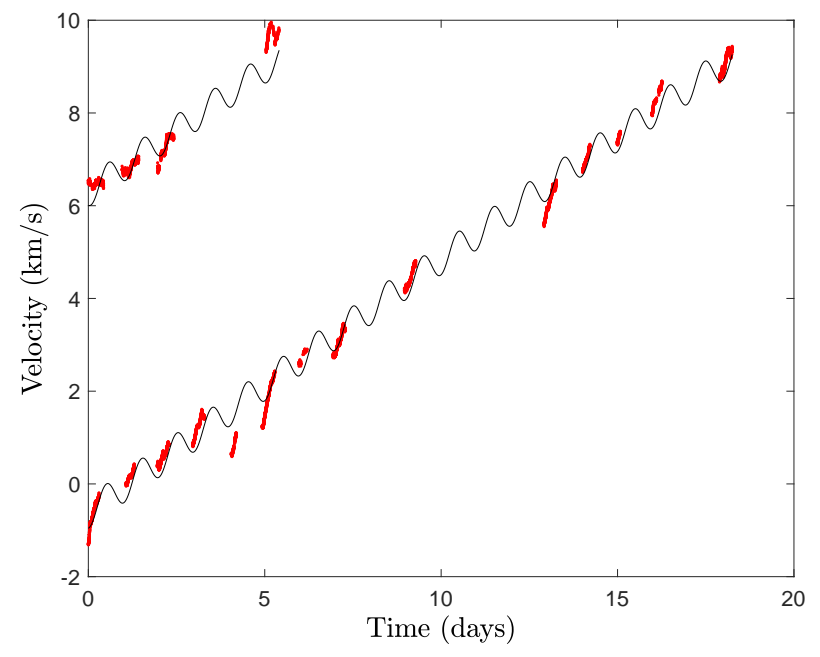

Figure 7: Time series of the mean radial-velocity of Jupiter for 2015 (top) and 2016 observations (bottom). Red dots are measured values and black curves theoretical radial-velocities from ephemeris.

PSF effect, as explained in equation B.1.

In order to estimate the stability of the residual maps, we measure the difference of velocity on four small areas of Jupiter's disk, near the north, south, east, and west edges of the planet. This reveals biases between north-south and east-west directions (Fig. 8). The biases include a large constant component in 2016 (mostly east-west), which was much smaller in 2015, and a variable component. The constant bias could be explained by an uncertainty about the pixel size on the sky. The variable part changes during the night in a relatively systematic way, which is correlated with the position of the telescope, and could have its origin on a slight modification of the instrumental phase maps during the night. One explanation is the evolution of the instrumental polarization when the multiple mirrors in the coude train of the telescope rotate. The rotation of Jupiter in the field could also produce this effect, if for example the instrumental phase map had a slight change with respect to the calibration file. In 2015, motions of the pupil could also account for this bias. It has to be noticed that the variations are much more systematic in 2016 than in 2015 . We explored possible causes of the observed effects with simulated data, but we could not identify a single origin, which means they might result from multiple causes.

To overcome these biases, it is necessary to rely on their apparent intrinsic spatial and temporal properties, which are very different from the structures expected for the zonal winds. We therefore empirically model and remove them as much as we can from the final product. We first remove the mean E-W gradient from each phase map (or from the stacked phase map, which is equivalent as all operations are linear). As said previously, the E-W gradient comes mainly from the difficulty to precisely know the size of the planet on the detector. As a consequence, we are unable to establish a precise rotation rate (reference frame for the rotation) and will 


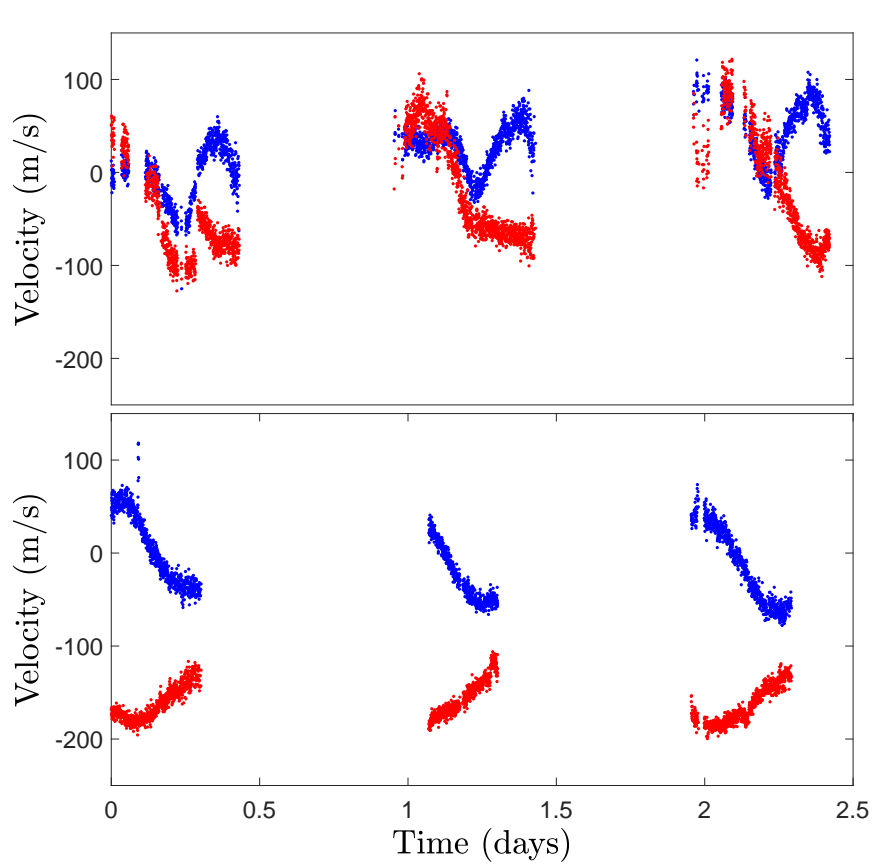

Figure 8: An illustration of the temporal evolution of the radial velocity maps. Blue lines indicate North-South differences, and red lines East-West difference, for the first three days of the 2015 (top) and 2016 (bottom) observing campaigns.

have to fix arbitrarily the zero of the wind velocity.

\subsection{Stacking the radial-velocity maps}

To increase the SNR of the radial-velocity maps, we compute one averaged phase-map for the full observing run, by weighting each map by the inverse of the photon noise of its corresponding image (i.e., individual weight per pixel for each image). We stacked together more than 4000 images in 2015 and more than 9000 in 2016. Figure 9 displays the stacked phase map for all the runs in 2015 (a) and 2016 , after removal of the mean $E-W$ gradient as explained in the previous section. We still note the presence of important trends that dominate the mean radial-velocity map and of some noisy rings. We also note horizontal structures that are compatible with radial velocities signature of zonal inds, as in the simulation of Fig. 5 in Doppler maps, prograde zonal winds will appear as an horizontal band with red in the west and blue in the east (colors reversed for retrograde winds), as we see here. The presence of rings is the effect of noise in the calibration map, which is used for all the images, and of the field rotation among the night. This high-frequency noise due to uncertainties in the calibration map is fixed on the detector, and therefore rotates when 800 all images are corrected from field rotation before stacking, producing the circular traces. These rings do not affect the values of the wind as they are washed out by the averaging along each latitude. The other trends are linked to instrumental bias, as discussed previously. We would like to 805

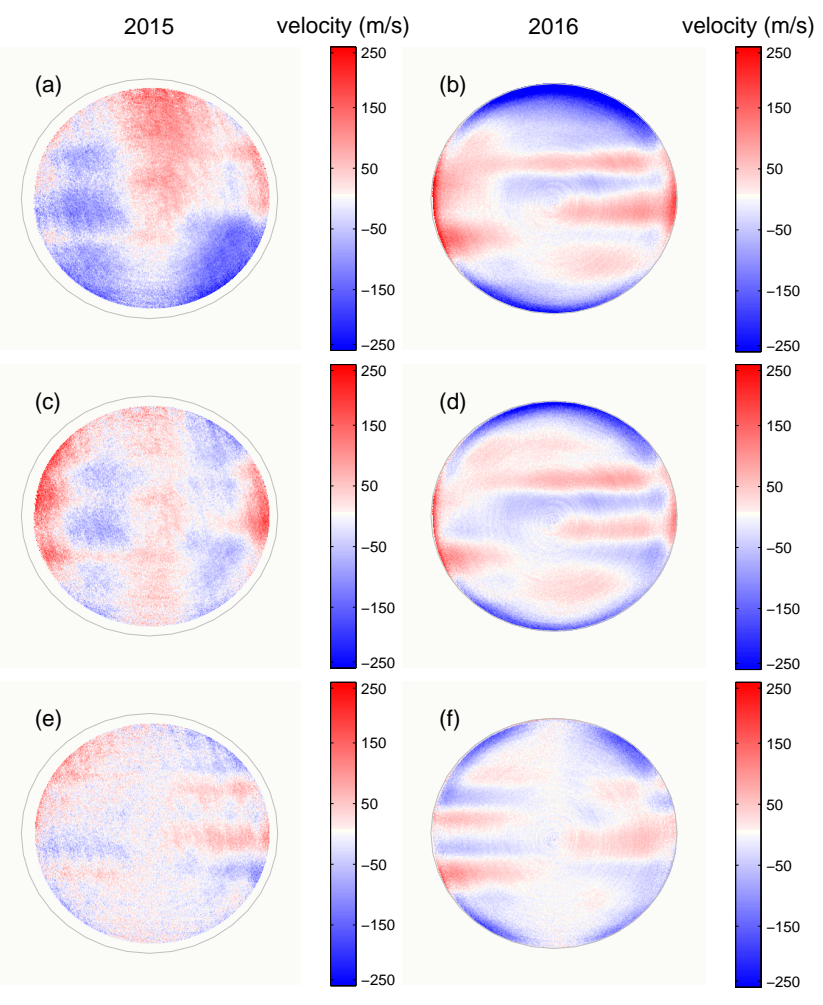

Figure 9: Mean radial-velocity maps of Jupiter obtained with JOVIAL-JIVE in 2015 (left) and 2016 (right). Top panels: sum of all of the radial-velocity maps $\tilde{v}_{\text {wind }}$ obtained for each campaign, once the solid-rotator contribution $\tilde{v}_{\mathrm{J}, \text { rot }}$ was subtracted from the original maps in 2015 (a) and 2016 (b). The smoother aspect of the 2016 map with respect to the 2015's is due to the larger amount of data acquired in 2016 and less noise. Middle panels: radial-velocity maps after correction of the quadratic bias described in Sect. 5.3 in 2015 (c) and 2016 (d). Bottom panels: same as the above, with mean Doppler shifts line by line removed, for 2015 (e) and 2016 (f). The area of Jupiter that is considered for radial-velocity measurements corresponds with the regions of intensity higher than $40 \%$ of the maximum flux, i.e., $90 \%$ of the planetary diameter in 2015 and above $10 \%$ of the maximum, corresponding to $99 \%$ of the diameter in 2016 (Jupiter's edge is indicated in gray).

remove them to produce clean maps and extract the wind profile. It has to be noticed that most of the biases that we see do not affect the calculation of the wind profile, except the twist of the map between Southern and Northern hemisphere, mainly visible in 2015.

To get rid of the bias, we model it with a 2-D second-order polynomial in the form:

$$
v_{p}(x, y)=p_{1} x+p_{2} x y+p_{3} y+p_{4} x^{2}+p 5 y^{2},
$$

weighted by the inverse of the theoretical photon noise $w$. We then subtract the 2-D polynomial fitting from the mean radialvelocity map to get a flattened map.

The filtering of such a second-order polynomial only affects the low spatial frequencies, and does not modify the shear structure of the zonal winds. Here, the term in $\mathbf{x}$ is null, as the rotation rate has been adjusted to the mean $E-W$ gradient, as said in previous section. In fact, only the term in xy affects the measured wind profile. The origin of this term is very likely due to changes in the sensitivity of the instru- 
ment during the night, related to a drift of the pupil inside the MZ (especially in 2015), simultaneously with the field rotation. Removing it does not affect the detail of the wind profile, but only the mean slope along the N-S axis. All the terms of correction are much lower in 2016 than in 2015, showing that most of the biases has been suppressed, probably thanks to the pupil stabilization.

The polynomial fitting is not able to remove all of the biases, especially those that display higher order structures in the spatial domain, as the central vertical band that we see particularly ${ }^{86}$ in the 2015 data. To get rid of the remaining spurious signals, we compute the projected radial-velocity along the $x$-axis by adding up the radial velocity along the vertical axis. The result is an average radial-velocity profile as a function of $x$. As for the polynomial fitting, the average profile is obtained by using ${ }^{870}$ weighted average:

$$
v_{\mathrm{EW}}(x)=\frac{\sum_{y}\left[v(x, y)-v_{p}(x, y)\right] w(x, y)}{\sum_{y} w(x, y)} .
$$

We then subtract line by line $v_{\mathrm{EW}}$ from the previously flattened velocity map(panels c and d of Fig. 9). This correction was ${ }^{875}$ not required in 2016, and not applied, as the map was already flat.

We also remove the mean velocity line by line. The mean Doppler shift line by line could be affected by bias but also contains information about possible vertical or meridional 880 wind components, if any. We do not aim at searching for such components in this work, therefore we just remove this mean value. The result is shown in the bottom panels e and $f$, where the value at the central meridian has been forced to zero, as in a pure zonal wind model. Again, these operations ${ }^{885}$ do not affect the zonal wind profile, as we will see below. We could have calculated a wind profile directly from the previous maps (c and d) with the same result, but this cleaning permits to see directly the zonal winds on the maps, as horizontal bands alternatively red shifted on the west wing and 890 blue shifted on the east wing for westward zonal winds, and reversed color for eastward winds.

\subsection{Average zonal wind profile}

If we assume the zonal wind to be invariant as function of 895 the longitude and that all observations were done at exact plan-

845 etary opposition, we can measure the zonal wind by fitting a 1-D polynomial line by line on the mean radial-velocity map (Fig. 9 bottom row). Indeed, a given rotation rate at a given latitude produces a linear $\mathbf{E}-\mathrm{W}$ slope on the Doppler 900 signal. Before doing so, it is necessary to correct our Doppler maps from the inclination of Jupiter's rotation axis with respect to the observer. In particular, in 2016, this angle was $\alpha=1.9^{\circ}$, which is close to its maximum value. Because of this inclination, a given parallel on Jupiter would appear as a curved ${ }_{905}$ line on the detector. The correction consists of interpolating the velocity maps on a new set of coordinates $(\tilde{x}, \tilde{y})$, where parallels becomes horizontal straight lines. In the new coordinate frame:

$$
\tilde{x}=x \quad \text { and } \quad \tilde{y}=R_{e q} \sin (\alpha+\beta)
$$

with :

$$
R_{e q}=\sqrt{R^{2}+\left(1-\mu^{2}\right) y^{2}} \text { and } \beta=\arctan \frac{y}{\sqrt{R^{2}-x^{2}-\mu^{2} y^{2}}}
$$

where $R$ is Jupiter's equatorial radius in pixels, which was 349 and 334 pixels in 2015 and 2016 respectively, $\mu$ is the ratio between the equatorial radius and the polar radius.

With this correction, the data are placed in the frame where the jovian rotation axis is vertical and the latitudes are horizontal. The zonal wind profile is then given by the slope of the velocity map. For the radial velocity of zonal wind, we approximately have $\tilde{v}_{\text {wind }}=\Omega_{d} x^{\prime}$ and thus $\Omega_{d}=\mathrm{d} v_{r} / \mathrm{d} x$, where $\Omega_{d}$ is the differential angular rotation (angular rotation without the solid rotation). The zonal wind $v_{\text {zon }}$ is then expressed as:

$$
v_{\text {zon }}=\frac{\mathrm{d} \tilde{v}_{\text {wind }}}{\mathrm{d} x} R_{e q} \cos \left(\lambda_{\mathrm{c}}\right)
$$

where $\lambda_{c}$ is the planetocentric latitude.

The value of $\mathrm{d} \tilde{v}_{\text {wind }} / \mathrm{d} x$ is obtained with a linear fit weighted by the inverse of the photon noise for each latitude. The resulting zonal velocity profile $\left(v_{\text {zon }}\right)$ is then smoothed over a window of 15 pixels, about the minimum size of the PSF.

The results for years 2015 and 2016 are shown in Fig. 11. where the Doppler wind profiles are compared to wind profile obtained from cloud tracking the same year (Johnson et al. 2018), and processed using the simulations explained in Sect. 4.5

We display two types of error bars on the wind profile on the figure 11. The smallest one is the result of the calculation of the photon noise level, by taking into account the true sensitivity and photon intensity at each latitude. It does not exceed $2 \mathrm{~m} \mathrm{~s}^{-1}$ in the central region. This error bar is much smaller in 2016 than in 2015, because the contrast of the fringes was higher and we observed for a much longer period. The largest error bar is the standard deviation of individual $\mathbf{1 5}$ minutes measurements night by night, averaged over the observation run. The mean error is about $20 \mathrm{~m} \mathrm{~s}^{-1}$, which is much larger than the corresponding photon noise. It includes some systematic variations of the bias along the night. It could also indicate genuine zonal winds variations with time and with longitude, as we measure a mean profile at different local times. At this stage, we do not intend to measure these variations and just provide a mean wind profile with its standard deviation, which overestimates the measurements error bars. Dispersion in the 2016 data appear similar or even larger than in $\mathbf{2 0 1 5}$, because many nights with variable atmospheric conditions were averaged.

These results are compared with zonal wind profiles obtained by cloud tracking from HST at similar epochs (01-19-2015 and 02-09-2016) in the visible domain at $502 \mathrm{~nm}$ (Johnson et al. 2018). For accurate comparison, we simulated radial velocity measurements corresponding to the cloud-tracking wind profile, taking into account the degradation by the PSF. In our simulations we use a Gaussian PSF which size is adjusted to correspond to the conditions of our radial velocity observations. The effect of the PSF over the shape of the profile is 


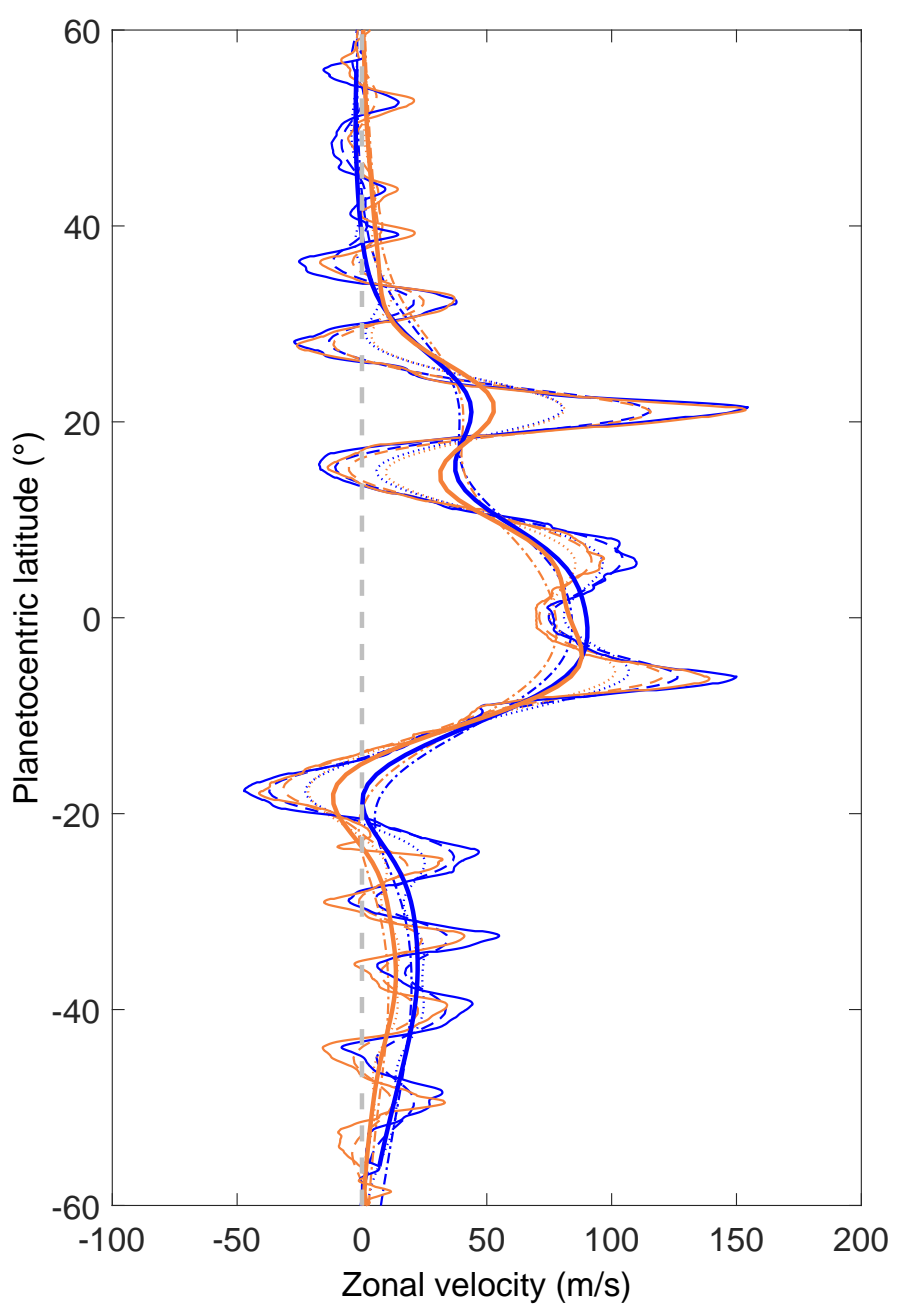

Figure 10: Simulated Doppler profile based on cloud-tracking profiles obtained in 2015 (blue) and 2016 (red) by Johnson et al. (2018) for different values of the PSF size, showing the degradation of the resolution. Plain lines are cloud-tracking profiles without degradation, dashed line corresponds to a PSF of 1 arcsec, dotted line is 2 arcsecs and dot-dashed is 4 arcsecs. The bold lines correspond to the average PSF of the DSI observations in 2015 (blue) and 2016 (red), with mean PSF of 3.9 and 3.0 arcsecs respectively

displayed in Fig. 10, where different values of the size of the PSF were applied. For comparison with the Doppler measurement, we calculated an average velocity map by adding simulated maps corresponding to each observations, using the same velocity profile from the cloud-tracking and the observed intensity map and the estimated PSF size. The average PSF size is about 3.9 arcsec in 2015, and about 3.0 in 2016.

Such a large PSF corresponds to about $5000 \mathrm{~km}$ at the center of Jupiter, or 4 degres in latitude at the equator, twice more at 60 degrees latitude. This resolution does not per-935 mit resolution of the smallest jet structures at high latitude. Overall, the Doppler measurements are in good agreement with cloud tracking (Fig. 11). However, we note a significant dis-

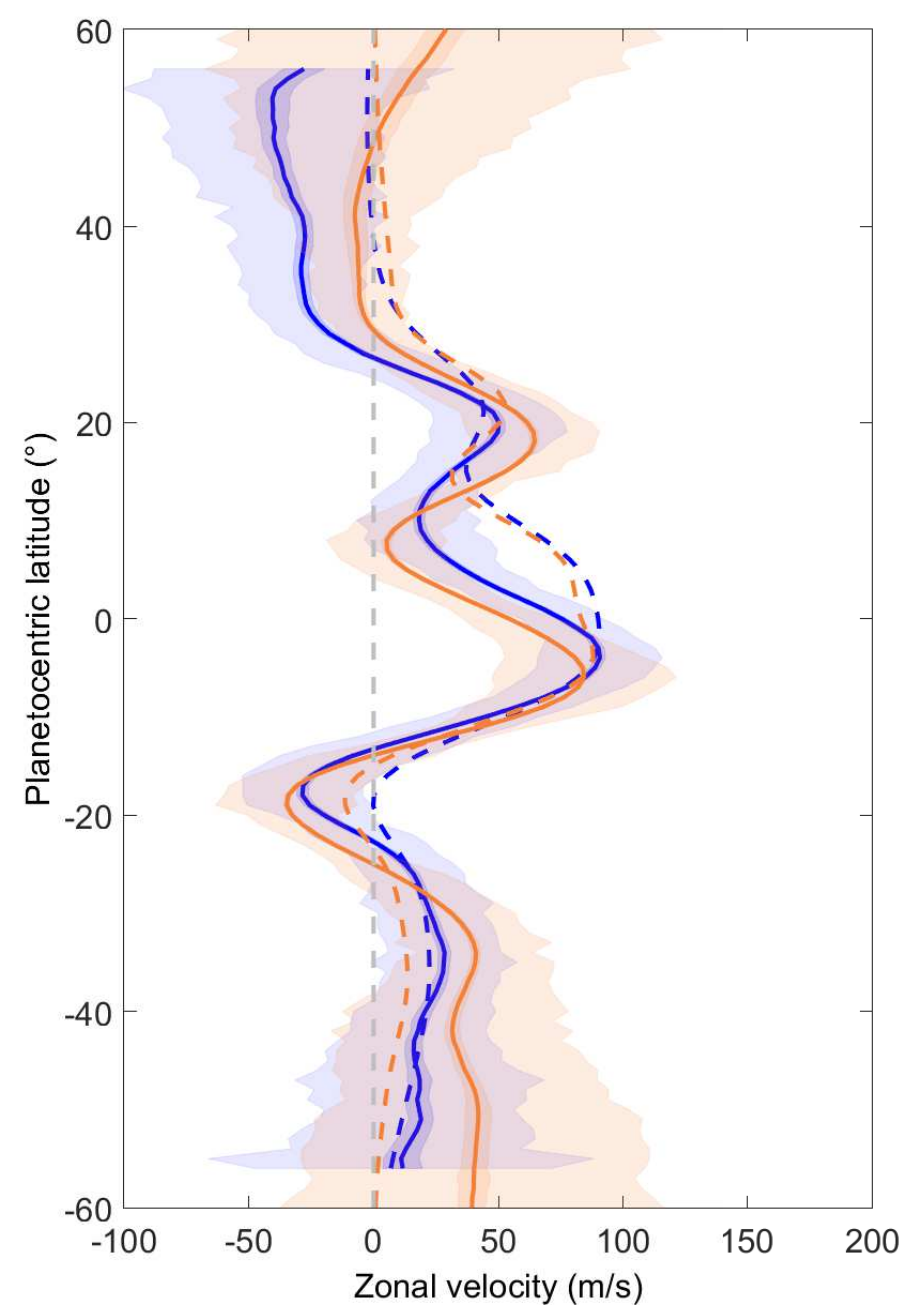

Figure 11: Mean zonal wind profile obtained by JOVIAL-JIVE in 2015 (blue) and 2016 (red). Darker shaded areas indicate the error bars resulting from photon noise only. Wider, less opaque areas indicate the standard deviation of velocity measurements. Dashed line: simulated Doppler profile deduced from cloud tracking performed by Johnson et al. (2018) with HST data of 2015 (blue) and 2016 (red) and then degraded by the observing conditions (pixel FOV, atmospheric seeing, optical distorsions, etc.) of the JOVIAL observations.

$\mathrm{N}$ where where zonal winds appear to be much slower than those derived from cloud tracking. We discuss this point in the next section.

\section{Discussion and prospects}

In this paper, we present the first Jovian zonal wind profiles obtained by Doppler shift measurements of reflected solar lines in the visible domain. Unlike cloud tracking, the Doppler shift supposedly reflects the true motion of the atmosphere at the level where the light is reflected. The novelty of this measurement and some of its results, such as the significant discrepancy between the radial velocity and the cloud tracked profiles around $5^{\circ} \mathrm{N}$, raise several questions.

The wind profiles shown in the previous section are obtained after removing large biases from the raw Doppler measure- 
${ }_{940}$ ments. It is therefore legitimate to wonder about the significance of the result. It has to be noticed that we are measuring winds of a few tenths of $\mathrm{m} \mathrm{s}^{-1}$, whereas the total rotation rate is larger than $24 \mathrm{~km} \mathrm{~s}^{-1}$ side by side. A small error of about $2 \%$ on the scale factor, i.e. the pixel size on the sky, could produce 945 the large East-West bias, as observed in 2016. This constant bias does not affect the wind profile, but only the absolute rotation rate, i.e. where the zero zonal wind line is in Fig. 11 .

Other variable biases are observed mainly on each side of 005 the velocity maps. They are averaged over each night. Sev950 eral physical reasons can explain the biases that we observe. Linear or quadratic biases may arise from uncertainties on the photometric or geometric calibrations, no better than a few percent, which alter the measurements mostly towards the edge of $f_{010}$ Jupiter. A part of the variable bias is clearly connected to the rotation of the telescope with respect to the instrument throughout the night. As previously mentioned, it likely results from the instrumental polarization related with the output polarizer cubes, which induces a leakage in the reflected beams that is 015 variable across the field. We plan to replace the cubes in the future. We also plan to test the possibility of depolarizing the beam at the entrance of the instrument. It has to be noticed that the varying bias is more systematic in 2016 , once the pupil was stabilized. Also, the remaining bias is mainly on the northern 020 and southern edges and is probably related to the intrinsic polarzation of the light at the poles of the planet. This means that we should be able to filter out more efficiently without altering the measurements. This is still the object of further investigation.

In brief, the bias that we filtered out, corresponding to the $e_{025}$ averaged bias over the night, is a low spatial-frequency signal, and does not affect the winds measurement at the relatively small-scale variations of the Jovian zones and belts. From an observational point of view, our results can be regarded as robust as they are consistent over two consecutive years, withoзо different instrumental settings and different telescopes configurations resulting in very different biases. The difference between the two yearly profiles lays within the error bars. However, these results are quite surprising as they are in opposition to cloud tracking measurements in the Northern equatorial band ${ }_{035}$ (between 0 and 15 degrees latitude).

It cannot be excluded that the discrepancies could come from an unindentified intrumental bias, although we didn't identify any plausible origin of such a bias. It should be noticed that our profiles are consistent with cloud-tracking profiles 040 elsewhere, in the southern hemisphere and above $15^{\circ} \mathrm{N}$. Infile stays within our error bars everywhere except in the northern equatorial band. The values found at latitudes higher than $40^{\circ}$ (either North or South) are less reliable, since the filtering ${ }_{045}$ of instrumental bias may affect the profile at the northern and southern edges, and they show stronger dispersion.

The region where the discrepancy arises is known to display peculiar properties with respect to other regions. First, among all latitudes, this region is the one that shows the most tem+050 poral and longitudinal variability according to cloud-tracking measurements (García-Melendo et al., 2011; Tollefson et al. 2017a; Johnson et al., 2018). This zone also has periodic hot spots (Allison, 1990; Showman and Ingersoll, 1998; Showman and Dowling, 2000). Previous studies interpreted the hot spots as the manifestation of thermal waves, i.e. Rossby wave modes propagating westward. Choi et al. (2013) pointed out that they could introduce a bias in the cloud tracking technique, by minimizing the velocity of the eastward wind. This effect is opposite to what we deduce from our Doppler measurements.

In situ measurements in a Jupiter hot spot by the Galileo probe yielded a wind velocity that increased from about $90 \mathrm{~m} \mathrm{~s}$ ${ }^{-1}$ near one bar (consistent with cloud tracking measurements) to $180 \mathrm{~m} \mathrm{~s}^{-1}$ at pressures deeper than 5 bars (Atkinson et al. 1998). We point out that it was a local measurement which does not necessarily reflect the mean wind velocity along the latitude, in particular because the probe entered in one of the hot-spot, which proved to be a peculiar region.

All of this suggests that particular processes are at work at this latitude, and that the velocities measured by cloud tracking and by Doppler measurements in this region do not necessary reflect the same physical displacement. We do not believe that the thermal activity in the northern equatorial zone could affect our Doppler shift measurements. Only solar lines are present in our narrow-band filter and they are not affected by the activity on Jupiter. The differences in brightness for the different latitudes do not seem to affect the velocity measurements, as proven by our results in the southern hemisphere.

The Doppler shift cannot distinguish different directions of the motion and could result from other components than a pure zonal wind. In Gaulme et al. (2018), we theoretically study how to retrieve the different components from Doppler maps taken at different rotation and phase angles. At the considered latitudes, the Doppler measurements are almost insensitive to a meridional motion if it would exist. Another option would be the existence of vertical motions, rapidly changing with the local hour on Jupiter, and affecting the mean radial velocity slope along the latitude. This seems very unrealistic as it would require vertical motions of the order of hundreds of $\mathrm{m} \mathrm{s}^{-1}$ to explain the discrepancies. Thus we conclude that our Doppler measurements reflect mainly the mean zonal motion of the cloud particles, and clearly shows a much slower prograde wind in the northern equatorial band, between 0 and 15 degrees latitudes, than what was previously measured by cloud-tracking.

We cannot verify the altitude of our measurements, as we integrate the reflected visible light at a given latitude. It might be possible that our measurements are dominated by the brightest features, which might have lower velocities if they are located at a higher level. However, as we observe in a very narrow band in the visible domain, we likely probe the same level as cloud-tracking measurements performed with a green filter. The only difference could come from a different weighting of regions with different velocities along the parallel. Our measurements were averaged over many days, so we expect that longitudinal variations should be washed out, except if these variations are function of the local time instead of the longitude.

Recent Juno results reveal that Jupiter's gravitational field is asymmetric (Iess et al., 2018), a consequence of zonal winds that extend about $3000 \mathrm{~km}$ deep (Kaspi et al., 2018; Guillot 
et al. 2018). The wind structure obtained from Juno's odd entional moments(Kaspi et al. 2018) is compatible with a simple wind profile fixed to the zonal wind versus latitude relation obtained by cloud tracking. However, the Galileo probe ${ }_{11}$ results (Bolton et al., 2017) indicate that the vertical structure of the zonal winds may be more complex. Our results can be forded as a new piece of the intriguing puzzle of the atmospheric dynamics of Jupiter.

At the moment, we cannot go further in the interpretation of the data. We presented several hypothesis (bias in cloudtracking measurement, different weighting of the motion by oud-tracking and Doppler, effect of other velocity compo $\overline{\overline{1}}_{115}$ nents), and cannot exclude a pure instrumental effect, although we carefully explored all possible bias and did not find a cause for such a difference. At this stage, we don't want to make a choice among these possibilities. More obervations are underway and planned in 2018. We expect to $\mathrm{Q}_{120}$ have less bias in the absolute phase measurement by minimizing the possible effects of the polarisation. In the future, we plan to improve the instrumental set-up in order to decrease the bias. In particular, the use of an Adaptive Optics system, already in of the PSF variations and distortions during the night. Our AO concept is derived from AO used for solar observations (see for instance Rimmele (2004)) which has proved to partially correct the atmospheric turbulence on the wavefield up to strated that a resolution of 0.5 arcsec on the whole surface of Jupiter could be achieved in moderate seeing conditions. Thanks to the imaging capability of our instrument, we also expect to study how the zonal winds change along the longitude on Jupiter.

\section{Acknowledgments}

The JOVIAL project is supported by the Agence Nationale de la Recherche under the contract number ANR-15-CE310014-01. JIVE in NM project is a NASA EPSCoR grant X14AN67A. The development of the Doppler Spectro Imager was partly supported by the Centre National d'Etudes Spatiales and by Thales Alenia Space. Ivan Gonçalves's $\mathrm{PhD}$ is granted by Observatoire de la Côte d'Azur, the JOVIAL project and the JIVE in NM project. Patrick Gaulme was supported in part by the German space agency (Deutsches Zentrum für Luftund Raumfahrt) under PLATO data grant 50OO1501. Observations would have not be possible without the valuable help of $f_{140}$ the MéO and C2PU teams at Calern observatory.

\section{Appendix A. Model of Jupiter}

Both a photometric and a radial-velocity models of Jupiter are needed for processing the data. As we see later on, a model of the photometric map of Jupiter is required to estimate the position of the planet on the detector, and to estimate the $a_{+145}$ plitude of image alteration caused by atmospheric turbulence above the observatory, known as atmospheric seeing. In addition, as mentioned in Eq. 16 , a radial velocity map of the solid-body rotation component of Jupiter is needed to compute $Z_{\text {wind }}$.

By taking into account the oblateness of Jupiter, the contour of its edge on the detector is an ellipse of semi-major axis equal to the equatorial radius $R_{\mathrm{eq}}$, and semi-minor axis $b^{\prime}$. Its value is deduced from calculating the tangent point of the ellipse with the line of sight (Fig. A.12):

$$
b^{\prime}=R_{\mathrm{eq}} \cos \alpha \sqrt{\tan ^{2} \alpha+\frac{1}{\mu^{2}}},
$$

where $\alpha$ is an axial tilt of its rotation with respect to the sky plane, and $\mu$ is the ratio between the equatorial and polar radii. We use the Yoder (1995) terminology, which estimates Jupiter's oblateness coefficient to be $f=0.0649$, such as $\mu=R_{\text {eq }} / R_{\text {pole }}=$ $(1-f)^{-1}$.

Here we consider the photometric profile of Jupiter's atmosphere in the visible to follow a Lambert law. The flux map inside the elliptic figure of Jupiter defined by Eq. A.1 is a function of the astronomical and physical parameters such as phase, radius, oblateness, and by assuming the Sun to be a point source. The coordinates used here are the same as in Fig. A.14 of the companion paper Gaulme et al. (2018). We note the coordinate $(x, y, z)$ with $(x, y)$ in the sky plane or CCD plane, and $\left(x^{\prime}, y^{\prime}, z^{\prime}\right)$ the natural coordinates to the ellipsoidal model with $z^{\prime}$ pointing to observer and $y^{\prime}$ to the north axis, $x^{\prime}$ is equal to $x$.

By associating the $(x, y)$ coordinates frame to the detector, the assumption of a Lambertian surface leads to the expression of the flux:

$$
I(x, y)=\vec{n} \cdot \vec{S}
$$

where $\vec{n}$ is the normal vector of ellipsoid surface of Jupiter, and $\vec{S}$ the source vector. In the case where $\alpha=0$ we have:

$$
\begin{gathered}
\vec{n}=\frac{1}{\sqrt{x^{\prime 2}+\mu^{4} y^{\prime 2}+z^{\prime 2}}}\left[\begin{array}{c}
x^{\prime} \\
\mu^{2} y^{\prime} \\
z^{\prime}
\end{array}\right] \\
\vec{S}=\left[\begin{array}{c}
\sin \varphi \\
0 \\
\cos \varphi
\end{array}\right]
\end{gathered}
$$

We can express $z^{\prime}$ according to $x^{\prime}$ and $y^{\prime}$ by:

$$
z^{\prime}\left(x^{\prime}, y^{\prime}\right)=\sqrt{R^{2}-x^{\prime 2}-\mu^{2} y^{\prime 2}},
$$

and therefore get:

$$
I(x, y)=\frac{x \sin \varphi+\sqrt{R^{2}-x^{2}-\mu^{2} y^{2}} \cos \varphi}{\sqrt{R^{2}+\mu^{2}\left(\mu^{2}-1\right) y^{2}}},
$$

where $\varphi$ is the phase angle, $R$ is the equatorial radius of Jupiter on the detector after dropping the "eq" subscript. Here, we neglect the tilt $\alpha$ of Jupiter with respect to the observer, which leads to a bias less than $0.3 \%$ in relative intensity. On top of this, we attempted to insert a texture map that reproduces the actual image of Jupiter, based on some public photometric maps of Jupiter, but it appeared to be useless in practice because there 


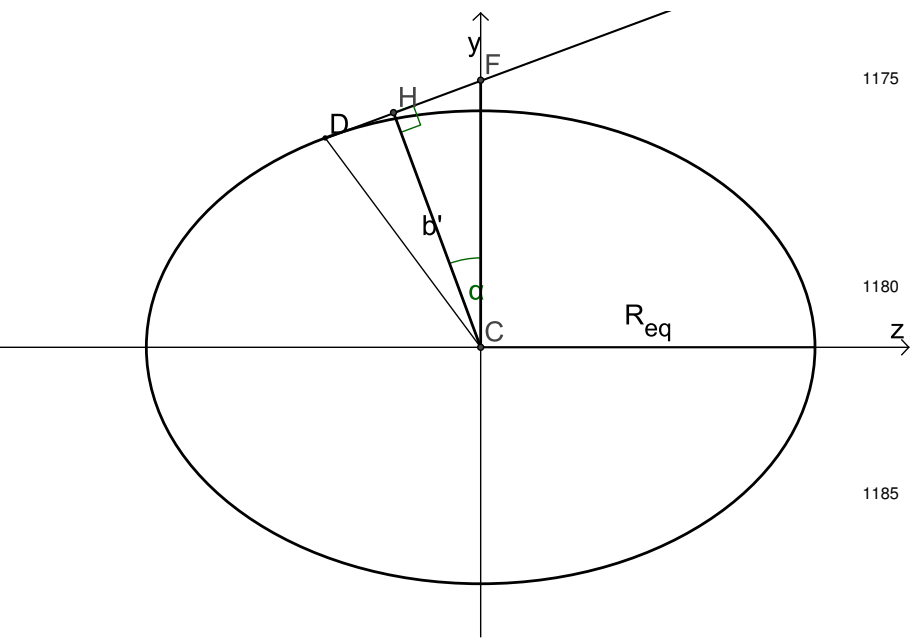

Figure A.12: Cut of the ellipsoid on the plane $(y, z)$. The line of sight is tangent to the ellipsoid in $D, b^{\prime}$ is the semi-minor axis of ellipse contour of Jupiter ${ }^{190}$ image. NOTE: I can barely see alpha in this figure. Also, what are C, F, H and $\mathrm{D}$ ?

is no such constant figure of Jupiter, as its atmospheric patterns evolve.

The radial velocity map associated with the solid-body component of Jupiter's rotation is a function of the phase angle $\varphi^{1195}$ the tilt of Jupiter's rotation axis toward the observer $\alpha$, and the oblateness $\mu$. For a null solar phase and tilt, the radial velocity towards the Sun is simply $v_{\odot}=\Omega x^{\prime}$, where $\Omega$ is the angular velocity and $x^{\prime}$ the abscissa along Jupiter's equator. With nonzero phase and axial tilt the radial velocity fields with respect to ${ }^{1200}$ the Sun $v_{\odot}$ and the observer $v_{\mathrm{O}}$ are respectively:

$$
v_{\odot}=\Omega \cos \alpha\left(x^{\prime} \cos \varphi-z^{\prime} \sin \varphi\right) \text { and } v_{\mathrm{O}}=\Omega x^{\prime} \cos \alpha .
$$

We neglected here the small latitude angle between the JupiterEarth direction and the Jupiter-Sun direction, which is less than $0.4^{\circ}$. We can express $z^{\prime}$ as a function of $x$ and $y$ :

$$
z^{\prime}(x, y)=N \sqrt{R^{2}-x^{2}-M y^{2}}-M y \sin \alpha
$$

with:

$$
M=\frac{\tan ^{2} \alpha+1}{\tan ^{2} \alpha+1 / \mu^{2}} \quad \text { and } \quad N=\frac{\cos \alpha}{\mu} \sqrt{M}
$$

By replacing $z^{\prime}$ in Eq. A.7 by Eq. A.8 and $x^{\prime}$ by $x$, and ${ }_{210}$ considering that $v=v_{\mathrm{O}}+v_{\odot}$, we get:

$$
\begin{array}{r}
v=\Omega \cos \alpha[(1+\cos \varphi) x- \\
\left.\left(N \sqrt{R^{2}-x^{2}-M y^{2}}-M y \sin \alpha\right) \sin \varphi\right] .
\end{array}
$$

Hence, a model of the complex phase map of solid rotation is defined by $Z_{\mathrm{J}, \text { rot }}=\exp \left(i S v_{\text {rot }}\right)$ where $v_{\text {rot }}=\left(v_{\mathrm{O}}+v_{\odot}\right) / 2$.

\section{Appendix B. Effect of the PSF}

The Point Spread Function (PSF) that convolves the images do not only degrade the resolution, but has other ef- fects in the measurement of Doppler velocities. We may note that one of the main expected difficulties in processing the data lays on the accuracy of estimating Jupiter's position, because 1 pixel, or 0.14 arcsec on the sky, corresponds to $75 \mathrm{~m} \mathrm{~s}^{-1}$. The determination of Jupiter's center as the barycenter of the image flux is sensitive to the transits of irregular and variable atmospheric patterns, as well as moons and their shadows. As explained in Gaulme et al. (2018), the determination of the edge of the image is also biased by the PSF. Therefore, we opted for a method based on fitting the position of the current acquisition image with the photometric image model degraded by the estimated atmospheric seeing at the time of acquisition.

The image PSF also affects the determination of the velocity, because of the simultaneous change of the intensity and the Doppler shift within the PSF, as explained in Gaulme et al. (2018). The effect is modeled by the equation:

$$
v(x, y)=\frac{\iint V(u, w) F(u, w) P(x-u, y-w) d u d w}{\iint F(u, v) P(x-u, y-w) d u d w}
$$

where $v$ is the actual radial-velocity map, $F$ is the actual photometric map and $P$ is the PSF of the atmospheric seeing.

Here, only the velocity term is convolved by the PSF. Actually, we should consider how the fringes are formed and calculate the effect of the PSF on the fringed images. Indeed, the fringes are not exactly a linear function of the velocity. Only their phase is linear. However, a complete simulation demonstrated that the expression in the above equation is an excellent approximation of the velocity bias. We will then use it both in the simulated data and to correct the velocity maps on Jupiter.

\section{Appendix C. Correction of the departure from quadrature}

As seen in Fig. 4, the phase shift between the four channels is not exactly $\pi / 2$. Therefore the quantities $U$ and $V$ calculated from the images through the equations 12 are not any more proportionnal to the $\cos$ and $\sin$ of the phase $\Phi$ as in equation 13, but also depends of the departure from quadrature $\epsilon$. This gives non orthogonal expressions for $U$ and $V$ :

$$
U=\gamma_{U} \cos (\Phi-\epsilon / 2) \quad \text { and } \quad V=\gamma_{V} \sin (\Phi+\epsilon / 2)
$$

where the phase departure $\epsilon$ has been balanced between both terms, or alternatively

$$
U_{n}=\cos (\Phi-\epsilon / 2) \quad \text { and } \quad V_{n}=\sin (\Phi+\epsilon / 2)
$$

where $U_{n}$ and $V_{n}$ are normalised by the contrast $\gamma_{U}$ and $\gamma_{V}$.

As proposed by Gaulme et al. (2008a), new orthogonal expressions can be obtained by applying the following operation:

$$
\begin{aligned}
U_{n}^{\prime} & =\frac{U_{n} \cos (\epsilon / 2)-V_{n} \sin (\epsilon / 2)}{\cos (\epsilon)} \\
V_{n}^{\prime} & =\frac{V_{n} \cos (\epsilon / 2)-U_{n} \sin (\epsilon / 2)}{\cos (\epsilon)}
\end{aligned}
$$



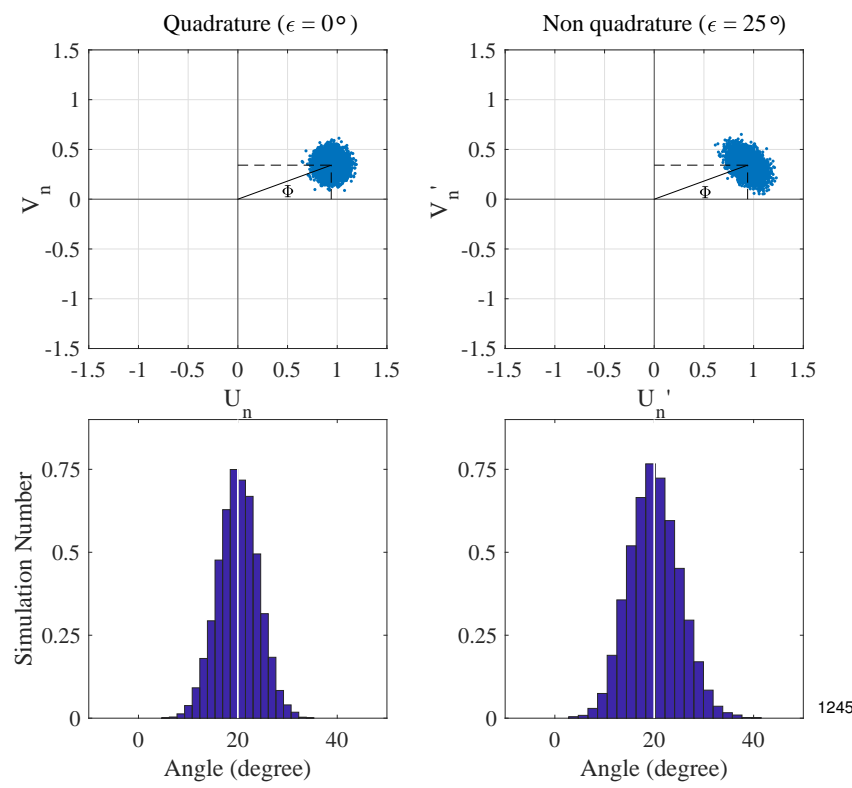

Figure C.13: Estimating the phase of the interferogram at a point of the image consists of computing $\arctan V_{n} / U_{n}$ in case of perfect quadrature, and $\arctan V_{n}^{\prime} / U_{n}^{\prime}$ when there is a departure to that condition. To illustrate the bias in measuring the phase once we correct the departure from quadrature, we consider 100,000 realizations of the interferogram, by producing $U_{n}$ and $V_{n}$ as normally random distributions around the average $\cos \Phi$ and $\sin \Phi$, respectively. In this simulation $\Phi=20^{\circ}, \gamma_{\mathrm{U}}=\gamma_{\mathrm{V}}=1$ (as $U_{n}$ and $V_{n}$ are normalized), and $\epsilon=25^{\circ}$. We then retrieve the phase $\Phi$ as $\arctan V_{n} / U_{n}$ (left) and $\arctan V_{n}^{\prime} / U_{n}^{\wedge 25}$ (right) and plot the histograms that correspond to both cases. In case of quadrature, the histogram is symmetrical around the true phase (vertical white line), whereas it is not in case of departure from quadrature.

The phase map can then be deduced from the argument of the 260 complex interferogram $Z^{\prime}=U^{\prime}+i V^{\prime}$.

In practice, computing $U^{\prime}$ and $V^{\prime}$ involves measuring the contrast $\gamma$ and the non-quadrature phase $\epsilon$. As indicated in the previous section, $\gamma_{i}$ is measured for each channel thanks to the instrumental interferogram calibration data. We note variations265 of the contrast across the field, which range from $4.5 \%$ at the center of the field down to $3 \%$ towards the edge. In the following, we use $\gamma_{U}=\left(\gamma_{1}+\gamma_{3}\right) / 2$ and $\gamma_{V}=\left(\gamma_{2}+\gamma_{4}\right) / 2$, which we use to normalize $U$ and $V$ (Eq. C.1). We estimate $\epsilon$ as the average value of the departure from phase quadrature:

$$
\epsilon=\frac{\Phi_{21}-\Phi_{43}-\pi}{2}
$$

where $\Phi_{i j}$ is the difference between the phase map calibration $\Phi_{i}$ and $\Phi_{j}$.

Finally, if transforming $U$ and $V$ into $U^{\prime}$ and $V^{\prime}$ allows us to process the data in the same way we would have done in case of perfect quadrature, it has the drawback of introducing spurious fringes. In the present paragraph, we explain why, and what to do to get rid of them. Let us consider a complex diagram where we plot $V$ as function of $U$, as done in Gaulme et al. (2008b). By considering the measurement errors on $U$ and $V$ to be equal, $\sigma_{\mathrm{U}}=\sigma_{\mathrm{V}}$, the error box around a point of values $\left(U_{i}, Y_{i}\right)$ is a disk of radius $\sigma_{\mathrm{U}}$ in the complex diagram (Fig C.13, left). In $\operatorname{such}_{285}$ a case, the error on the phase $\Phi=\arctan V / U$ is symmetrical
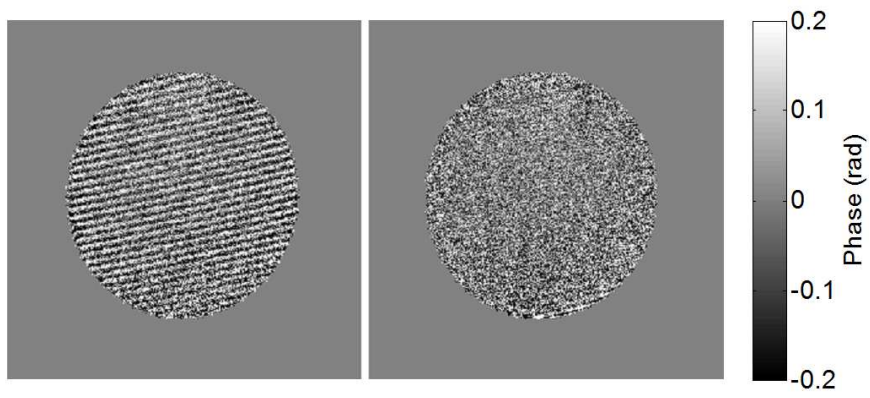

Figure C.14: Left: periodic bias of phase with cumulative 50 images. Right: correction after model fitting (simulation).

around its average, which makes $\arctan V / U$ an unbiased estimator of the phase. To the contrary, once we transform $(U, V)$ into $\left(U^{\prime}, V^{\prime}\right)$, the correlation of $U^{\prime}$ and $V^{\prime}$ make their errors correlated, and the error area is no more a disk but an ellipse tilted by $45^{\circ}$. In such a case, the error on $\Phi^{\prime}=\arctan V^{\prime} / U^{\prime}$ is no more symmetrical around the estimator, so that $\arctan V^{\prime} / U^{\prime}$ turns out to be a biased estimator of $\Phi^{\prime}$. Since the orientation of the ellipse is independent from the value of the phase, the bias on the phase is the same for $\Phi^{\prime}$ and $\Phi^{\prime}+\pi$, which means that the bias is periodic with a with a frequency twice that of the fringes. It can be seen also that the dispersion is increased by a factor $\frac{1}{\cos (\epsilon / 2)}$

We thus have a bias which is presented as a quasi-sinusoidal modulation with a frequency twice that of the fringes. The amplitude of the periodic bias is only a fraction of the phase noise, so the bias will appear after the accumulation of several phase maps. It can be suppressed in large part, by applying a correction of a model of the bias obtained by a fit of the amplitude.

We checked by Monte Carlo simulation that the application of this model correctly removes the spurious fringes if the noise is small enough and if the departure to quadrature remains below 30 degrees, which is our case here. The possible remaining fringes can be eliminated by filtering the specific spatial frequency.

\section{References}

Allison, M., Feb. 1990. Planetary waves in Jupiter's equatorial atmosphere. Icarus83, 282-307.

Appourchaux, T., Grundahl, T., 2015. Asteroseismology observations and space missions. Extraterrestrial Seismology, Cambridge: Cambridge University Press, pp. 189-202.

Asay-Davis, X. S., Marcus, P. S., Wong, M. H., de Pater, I., Sep. 2009. Jupiter's shrinking Great Red Spot and steady Oval BA: Velocity measurements with the Advection Corrected Correlation Image Velocimetry automated cloudtracking method. Icarus203, 164-188.

Atkinson, D. H., Pollack, J. B., Seiff, A., Sep. 1998. The Galileo probe Doppler wind experiment: Measurement of the deep zonal winds on Jupiter. JGR103, 22911-22928.

Barrado-Izagirre, N., Rojas, J. F., Hueso, R., Sánchez-Lavega, A., Colas, F., Dauvergne, J. L., Peach, D., IOPW Team, Jun. 2013. Jupiter's zonal winds and their variability studied with small-size telescopes. A\&A554, A74.

Beebe, R., 1994. Jupiter : the giant planet.

Bolton, S. J., Adriani, A., Adumitroaie, V., Allison, M., Anderson, J., Atreya, S., Bloxham, J., Brown, S., Connerney, J. E. P., DeJong, E., Folkner, W., 
Gautier, D., Grassi, D., Gulkis, S., Guillot, T., Hansen, C., Hubbard, W. B., Iess, L., Ingersoll, A., Janssen, M., Jorgensen, J., Kaspi, Y., Levin, S. M., Li, C., Lunine, J., Miguel, Y., Mura, A., Orton, G., Owen, T., Ravine, M., Smith1360 E., Steffes, P., Stone, E., Stevenson, D., Thorne, R., Waite, J., Durante, D., Ebert, R. W., Greathouse, T. K., Hue, V., Parisi, M., Szalay, J. R., Wilson, R., May 2017. Jupiter's interior and deep atmosphere: The initial pole-to-pole passes with the Juno spacecraft. Science 356, 821-825.

Brogi, M., de Kok, R. J., Albrecht, S., Snellen, I. A. G., Birkby, J. L., Schwarzł365 H., Feb. 2016. Rotation and Winds of Exoplanet HD 189733 b Measured with High-dispersion Transmission Spectroscopy. ApJ817, 106.

Bundy, K., Bershady, M. A., Law, D. R., Yan, R., Drory, N., MacDonald, N., Wake, D. A., Cherinka, B., Sánchez-Gallego, J. R., Weijmans, A.-M., Thomas, D., Tremonti, C., Masters, K., Coccato, L., Diamond-Stanic, A. M.1370 Aragón-Salamanca, A., Avila-Reese, V., Badenes, C., Falcón-Barroso, J., Belfiore, F., Bizyaev, D., Blanc, G. A., Bland-Hawthorn, J., Blanton, M. R., Brownstein, J. R., Byler, N., Cappellari, M., Conroy, C., Dutton, A. A., Emsellem, E., Etherington, J., Frinchaboy, P. M., Fu, H., Gunn, J. E., Harding, P., Johnston, E. J., Kauffmann, G., Kinemuchi, K., Klaene, M. A.1375 Knapen, J. H., Leauthaud, A., Li, C., Lin, L., Maiolino, R., Malanushenko, V., Malanushenko, E., Mao, S., Maraston, C., McDermid, R. M., Merrifield, M. R., Nichol, R. C., Oravetz, D., Pan, K., Parejko, J. K., Sanchez, S. F., Schlegel, D., Simmons, A., Steele, O., Steinmetz, M., Thanjavur, K., Thompson, B. A., Tinker, J. L., van den Bosch, R. C. E., Westfall, K. B.1380 Wilkinson, D., Wright, S., Xiao, T., Zhang, K., Jan. 2015. Overview of the SDSS-IV MaNGA Survey: Mapping nearby Galaxies at Apache Point Observatory. ApJ798, 7.

Cacciani, A., Dolci, M., Moretti, P. F., D’Alessio, F., Giuliani, C., Micolucci, E., Di Cianno, A., Jun. 2001. Search for global oscillations on Jupiter with 385 a double-cell sodium magneto-optical filter. A\&A372, 317-325.

Choi, D. S., Banfield, D., Gierasch, P., Showman, A. P., May 2007. Velocity and vorticity measurements of Jupiter's Great Red Spot using automated cloud feature tracking. Icarus $188,35-46$.

Choi, D. S., Showman, A. P., Dec. 2011. Power spectral analysis of Jupiterśs 39 clouds and kinetic energy from Cassini. Icarus216, 597-609.

Choi, D. S., Showman, A. P., Vasavada, A. R., Simon-Miller, A. A., Apr. 2013. Meteorology of Jupiter's equatorial hot spots and plumes from Cassini. Icarus223, 832-843.

Clancy, R. T., Sandor, B. J., Moriarty-Schieven, G., Feb. 2012. Circulation of 395 the Venus upper mesosphere/lower thermosphere: Doppler wind measurements from 2001-2009 inferior conjunction, sub-millimeter CO absorption line observations. Icarus $217,794-812$.

Clancy, R. T., Sandor, B. J., Moriarty-Schieven, G. H., Aug. 2008. Venus upper atmospheric $\mathrm{CO}$, temperature, and winds across the afternoon/evening 400 terminator from June 2007 JCMT sub-millimeter line observations. PSS56, 1344-1354.

Deming, D., Reuter, D., Jennings, D., Bjoraker, G., McCabe, G., Fast, K., Wiedemann, G., Apr. 1997. Observations and Analysis of Longitudinal Thermal Waves on Jupiter. Icarus126, 301-312.

Flasar, F. M., Kunde, V. G., Achterberg, R. K., Conrath, B. J., Simon-Miller, A. A., Nixon, C. A., Gierasch, P. J., Romani, P. N., Bézard, B., Irwin, P., Bjoraker, G. L., Brasunas, J. C., Jennings, D. E., Pearl, J. C., Smith, M. D., Orton, G. S., Spilker, L. J., Carlson, R., Calcutt, S. B., Read, P. L., Taylor, F. W., Parrish, P., Barucci, A., Courtin, R., Coustenis, A., Gautier, D., Lel ${ }_{4410}$ louch, E., Marten, A., Prangé, R., Biraud, Y., Fouchet, T., Ferrari, C., Owen, T. C., Abbas, M. M., Samuelson, R. E., Raulin, F., Ade, P., Césarsky, C. J., Grossman, K. U., Coradini, A., Jan. 2004. An intense stratospheric jet on Jupiter. Nature427, 132-135.

Fletcher, L. N., Greathouse, T. K., Orton, G. S., Sinclair, J. A., Giles, R. S.1415 Irwin, P. G. J., Encrenaz, T., Nov. 2016. Mid-infrared mapping of Jupiter's temperatures, aerosol opacity and chemical distributions with IRTF/TEXES. Icarus 278, 128-161

Gabsi, Y., Bertaux, J. L., Hauchecorne, A., Schmitt, J., Guibert, S., Oct. 2008. Measuring Venus' winds using the Absolute Astronomical Accelerometer1420 Solid super-rotation model of Venus' clouds. PSS56, 1454-1466.

García-Melendo, E., Arregi, J., Rojas, J. F., Hueso, R., Barrado-Izagirre, N., Gómez-Forrellad, J. M., Pérez-Hoyos, S., Sanz-Requena, J. F., SánchezLavega, A., Feb. 2011. Dynamics of Jupiter's equatorial region at cloud top level from Cassini and HST images. Icarus211, 1242-1257.

1355 García-Melendo, E., Sánchez-Lavega, A., Aug. 2001. A Study of the Stability of Jovian Zonal Winds from HST Images: 1995-2000. Icarus152, 316-330.

Gaulme, P., Schmider, F.-X., Gay, J., Guillot, T., Jacob, C., Jul. 2011. Detection of Jovian seismic waves: a new probe of its interior structure. A\&A531, A104.

Gaulme, P., Schmider, F. X., Gay, J., Jacob, C., Alvarez, M., Reyes-Ruiz, M., Belmonte, J. A., Fossat, E., Jeanneaux, F., Valtier, J.-C., Nov. 2008a. SYMPA, a dedicated instrument for Jovian seismology. II. Real performance and first results. A\&A490, 859-871.

Gaulme, P., Schmider, F.-X., Goncalves, I., Apr. 2018. Measuring Planetary Atmospheric Dynamics with Doppler Spectroscopy. ArXiv e-prints.

Gaulme, P., Schmider, F.-X., Grec, C., López Ariste, A., Widemann, T., Gelly, B., Aug. 2008b. Venus wind map at cloud top level with the MTR/THEMIS visible spectrometer, I: Instrumental performance and first results. PSS56, 1335-1343.

Gonçalves, I., Schmider, F.-X., Bresson, Y., Dejonghe, J., Preis, O., RobbeDubois, S., Appourchaux, T., Boumier, P., Leclec'h, J.-C., Morinaud, G., Gaulme, P., Jackiewicz, J., Aug. 2016. Advances in the development of a Mach-Zehnder interferometric Doppler imager for seismology of giant planets. In: Ground-based and Airborne Instrumentation for Astronomy VI. Vol. 9908. p. 99083M.

Guillot, T., Miguel, Y., Militzer, B., Hubbard, W. B., Kaspi, Y., Galanti, E., Cao, H., Helled, R., Wahl, S. M., Iess, L., Folkner, W. M., Stevenson, D. J., Lunine, J. I., Reese, D. R., Biekman, A., Parisi, M., Durante, D., Connerney, J. E. P., Levin, S. M., Bolton, S. J., Mar. 2018. A suppression of differential rotation in Jupiterś deep interior. Nature555, 227-230.

Iess, L., Folkner, W. M., Durante, D., Parisi, M., Kaspi, Y., Galanti, E., Guillot, T., Hubbard, W. B., Stevenson, D. J., Anderson, J. D., Buccino, D. R., Casajus, L. G., Milani, A., Park, R., Racioppa, P., Serra, D., Tortora, P., Zannoni, M., Cao, H., Helled, R., Lunine, J. I., Miguel, Y., Militzer, B., Wahl, S., Connerney, J. E. P., Levin, S. M., Bolton, S. J., Mar. 2018. Measurement of Jupiterś asymmetric gravity field. Nature555, 220-222.

Ingersoll, A. P., Dowling, T. E., Gierasch, P. J., Orton, G. S., Read, P. L., Sánchez-Lavega, A., Showman, A. P., Simon-Miller, A. A., Vasavada, A. R., 2004. Dynamics of Jupiter's atmosphere. pp. 105-128.

Johnson, P. E., Morales-Juberías, R., Simon, A., Gaulme, P., Wong, M. H., Cosentino, R. G., Jun. 2018. Longitudinal variability in Jupiter's zonal winds derived from multi-wavelength HST observations. PSS155, 2-11.

Kaspi, Y., Galanti, E., Hubbard, W. B., Stevenson, D. J., Bolton, S. J., Iess, L., Guillot, T., Bloxham, J., Connerney, J. E. P., Cao, H., Durante, D., Folkner, W. M., Helled, R., Ingersoll, A. P., Levin, S. M., Lunine, J. I., Miguel, Y., Militzer, B., Parisi, M., Wahl, S. M., Mar. 2018. Jupiterś atmospheric jet streams extend thousands of kilometres deep. Nature555, 223-226.

Kostiuk, T., Fast, K. E., Livengood, T. A., Hewagama, T., Goldstein, J. J., Espenak, F., Buhl, D., 2001. Direct measurement of winds on Titan. GRL28, 2361-2364

Kostiuk, T., Hewagama, T., Fast, K. E., Livengood, T. A., Annen, J., Buhl, D., Sonnabend, G., Schmülling, F., Delgado, J. D., Achterberg, R., Nov. 2010. High spectral resolution infrared studies of Titan: Winds, temperature, and composition. PSS58, 1715-1723.

Kostiuk, T., Livengood, T. A., Hewagama, T., Sonnabend, G., Fast, K. E., Murakawa, K., Tokunaga, A. T., Annen, J., Buhl, D., Schmülling, F., Nov. 2005. Titan's stratospheric zonal wind, temperature, and ethane abundance a year prior to Huygens insertion. GRL32, L22205.

Kostiuk, T., Livengood, T. A., Sonnabend, G., Fast, K. E., Hewagama, T., Murakawa, K., Tokunaga, A. T., Annen, J., Buhl, D., Schmülling, F., Luz, D., Witasse, O., Jul. 2006. Stratospheric global winds on Titan at the time of Huygens descent. Journal of Geophysical Research (Planets) 111, E07S03.

Lellouch, E., Rosenqvist, J., Goldstein, J. J., Bougher, S. W., Paubert, G., Dec. 1991. First absolute wind measurements in the middle atmosphere of Mars. ApJ383, 401-406.

Lellouch, E., Witasse, O., Aug. 2008. A coordinated campaign of Venus ground-based observations and Venus Express measurements. PSS56, 1317-1319.

Li, L., Gierasch, P. J., Achterberg, R. K., Conrath, B. J., Flasar, F. M., Vasavada, A. R., Ingersoll, A. P., Banfield, D., Simon-Miller, A. A., Fletcher, L. N., Dec. 2008. Strong jet and a new thermal wave in Saturn's equatorial stratosphere. GRL35, L23208.

Li, L., Ingersoll, A. P., Vasavada, A. R., Porco, C. C., Del Genio, A. D., Ewald, S. P., Nov. 2004. Life cycles of spots on Jupiter from Cassini images. Icarus172, 9-23.

Limaye, S. S., Mar. 1986. Jupiter - New estimates of the mean zonal flow at the cloud level. Icarus65, 335-352.

Louden, T., Wheatley, P. J., Dec. 2015. Spatially Resolved Eastward Winds and 
Rotation of HD 189733b. ApJL814, L24.

Mac Low, M.-M., Ingersoll, A. P., Mar. 1986. Merging of vortices in the atmosphere of Jupiter - an analysis of Voyager images. Icarus65, 353-369.

Machado, P., Luz, D., Widemann, T., Lellouch, E., Witasse, O., Sep. 2012. Mapping zonal winds at Venus' cloud tops from ground-based Doppler velocimetry. Icarus221, 248-261

Machado, P., Widemann, T., Luz, D., Peralta, J., Nov. 2014. Wind circulation regimes at Venus' cloud tops: Ground-based Doppler velocimetry using CFHT/ESPaDOnS and comparison with simultaneous cloud tracking measurements using VEx/VIRTIS in February 2011. Icarus243, 249-263.

Machado, P., Widemann, T., Peralta, J., Gonçalves, R., Donati, J.-F., Luz1510 D., Mar. 2017. Venus cloud-tracked and doppler velocimetry winds from CFHT/ESPaDOnS and Venus Express/VIRTIS in April 2014. Icarus285, 826.

Mitchell, J. L., Beebe, R. F., Ingersoll, A. P., Garneau, G. W., Sep. 1981. Flow fields within Jupiter's Great Red SPOT and White Oval BC. JGR86, 8751-515 8757.

Morales-Juberías, R., Sánchez-Lavega, A., Lecacheux, J., Colas, F., May 2002a. A Comparative Study of Jovian Anticyclone Properties from a SixYear (1994-2000) Survey. Icarus157, 76-90.

Morales-Juberías, R., Sánchez-Lavega, A., Lecacheux, J., Colas, F., Dec1520 2002b. A Comparative Study of Jovian Cyclonic Features from a Six-Year (1994-2000) Survey. Icarus160, 325-335.

Moreno, R., Lellouch, E., Forget, F., Encrenaz, T., Guilloteau, S., Millour, E., Jun. 2009. Wind measurements in Mars' middle atmosphere: IRAM Plateau de Bure interferometric CO observations. Icarus201, 549-563.

Moreno, R., Marten, A., Hidayat, T., Jul. 2005. Interferometric measurements of zonal winds on Titan. A\&A437, 319-328.

Mosser, B., Mekarnia, D., Maillard, J. P., Gay, J., Gautier, D., Delache, P., Jan. 1993. Seismological observations with a Fourier transform spectrometer Detection of Jovian oscillations. A\&A267, 604-622.

Moullet, A., Lellouch, E., Moreno, R., Gurwell, M., Sagawa, H., Oct. 2012. Wind mapping in Venus' upper mesosphere with the IRAM-Plateau de Bure interferometer. A\&A546, A102.

Owen, C. B., Makedon, F., Nov. 1996. High Quality Alias Free Image Rotation. In: Conference Record of The Thirtieth Asilomar Conference on Signals 1535 Systems and Computers. Vol. 1. Dartmouth College, Computer Science, pp. 115-119.

Porco, C. C., West, R. A., McEwen, A., Del Genio, A. D., Ingersoll, A. P., Thomas, P., Squyres, S., Dones, L., Murray, C. D., Johnson, T. V., Burns, J. A., Brahic, A., Neukum, G., Veverka, J., Barbara, J. M., Denk, T., Evans\$1540 M., Ferrier, J. J., Geissler, P., Helfenstein, P., Roatsch, T., Throop, H., Tiscareno, M., Vasavada, A. R., Mar. 2003. Cassini Imaging of Jupiter's Atmosphere, Satellites, and Rings. Science 299, 1541-1547.

Reuter, D. C., Simon-Miller, A. A., Lunsford, A., Baines, K. H., Cheng, A. F., Jennings, D. E., Olkin, C. B., Spencer, J. R., Stern, S. A., Weaver, H. A.1545 Young, L. A., Oct. 2007. Jupiter Cloud Composition, Stratification, Convection, and Wave Motion: A View from New Horizons. Science 318, 223

Rimmele, T. R., Apr. 2004. Plasma Flows Observed in Magnetic Flux Concentrations and Sunspot Fine Structure Using Adaptive Optics. ApJ604, 906923

Schmider, F. X., Appourchaux, T., Gaulme, P., Guillot, T., Sato, B., Murphy, N., Daban, J. B., Gay, J., Soulat, L., Baudin, F., Boumier, P., Ollivier, M., Bordé. P., Jackiewicz, J., Ida, S., Showman, A. P., Dec. 2013. The JOVIAL Project for Jovian Seismology. In: Fifty Years of Seismology of the Sun and Stars. Vol. 478. p. 119

Schmider, F.-X., Fossat, E., Mosser, B., Aug. 1991. Possible detection of Jovian global oscillations. A\&A248, 281-291.

Schmider, F. X., Gay, J., Gaulme, P., Jacob, C., Abe, L., Alvarez, M., Belmonte, J. A., Fossat, E., Gelly, B., Guillot, T., Jeanneaux, F., Mosser, B., Valtier, J.-C., Nov. 2007. SYMPA, a dedicated instrument for Jovian seismology. I. Principle and performance. A\&A474, 1073-1080.

Showman, A. P., Dowling, T. E., Sep. 2000. Nonlinear Simulations of Jupiter's 5-Micron Hot Spots. Science 289, 1737-1740.

Showman, A. P., Ingersoll, A. P., Apr. 1998. Interpretation of Galileo Probe Data and Implications for Jupiter's Dry Downdrafts. Icarus 132, 205-220.

000

Simon-Miller, A. A., Rogers, J. H., Gierasch, P. J., Choi, D., Allison, M. D., Adamoli, G., Mettig, H.-J., Apr. 2012. Longitudinal variation and waves in Jupiter's south equatorial wind jet. Icarus218, 817-830.

Snellen, I. A. G., Brandl, B. R., de Kok, R. J., Brogi, M., Birkby, J., Schwarz, H., May 2014. Fast spin of the young extrasolar planet $\beta$ Pictoris b. Nature 509, 63-65.

Sornig, M., Livengood, T., Sonnabend, G., Kroetz, P., Stupar, D., Kostiuk, T., Schieder, R., Aug. 2008. Venus upper atmosphere winds from ground-based heterodyne spectroscopy of $\mathrm{CO}_{2}$ at 10 $\mu$ m wavelength. PSS56, 1399-1406.

Sornig, M., Livengood, T. A., Sonnabend, G., Stupar, D., Kroetz, P., Feb. 2012. Direct wind measurements from November 2007 in Venus' upper atmosphere using ground-based heterodyne spectroscopy of $\mathrm{CO}_{2}$ at $10 \mu \mathrm{m}$ wavelength. Icarus217, 863-874.

Soulat, L., Schmider, F.-X., Robbe-Dubois, S., Appourchaux, T., Bresson, Y., Daban, J.-B., Gaulme, P., Gay, J., Gouvret, C., Sep. 2012. Echoes: a new instrumental concept of spectro-imaging for Jovian seismology. In: Space Telescopes and Instrumentation 2012: Optical, Infrared, and Millimeter Wave. Vol. 8442 of Proc. SPIE. p. 84424M.

Stamp, A. P., Dowling, T. E., Oct. 1993. Jupiter's winds and Arnol'd's second stability theorem: Slowly moving waves and neutral stability. JGR98, 18.

Tollefson, J., Wong, M. H., de Pater, I., Simon, A. A., Orton, G. S., Rogers, J. H., Atreya, S. K., Cosentino, R. G., Januszewski, W., Morales-Juberías, R., Marcus, P. S., Nov. 2017a. Changes in Jupiter's Zonal Wind Profile preceding and during the Juno mission. Icarus 296, 163-178.

Tollefson, J., Wong, M. H., Pater, I. d., Simon, A. A., Orton, G. S., Rogers, J. H., Atreya, S. K., Cosentino, R. G., Januszewski, W., Morales-Juberías, R., Marcus, P. S., Nov. 2017b. Changes in Jupiter's Zonal Wind Profile preceding and during the Juno mission. Icarus296, 163-178.

Traub, W. A., Carleton, N. P., Jun. 1975. Spectroscopic observations of winds on Venus. Journal of Atmospheric Sciences 32, 1045-1059.

Underwood, T. A., Voelz, D., Schmider, F.-X., Jackiewicz, J., Dejonghe, J., Bresson, Y., Hull, R., Goncalves, I., Gualme, P., Morand, F., Preis, O., Sep. 2017. Adaptation of Dunn Solar Telescope for Jovian Doppler spectro imaging. In: Society of Photo-Optical Instrumentation Engineers (SPIE) Conference Series. Vol. 10401 of Society of Photo-Optical Instrumentation Engineers (SPIE) Conference Series. p. 104010Y.

Vasavada, A. R., Ingersoll, A. P., Banfield, D., Bell, M., Gierasch, P. J., Belton, M. J. S., Orton, G. S., Klaasen, K. P., DeJong, E., Breneman, H. H., Jones, T. J., Kaufman, J. M., Magee, K. P., Senske, D. A., Sep. 1998. Galileo Imaging of Jupiter's Atmosphere: The Great Red Spot, Equatorial Region, and White Ovals. Icarus135, 265-275.

Widemann, T., Lellouch, E., Campargue, A., Oct. 2007. New wind measurements in Venus lower mesosphere from visible spectroscopy. PSS55, 17411756

Widemann, T., Lellouch, E., Donati, J.-F., Aug. 2008. Venus Doppler winds at cloud tops observed with ESPaDOnS at CFHT. PSS56, 1320-1334.

Wyant, J. C., Nov. 1975. Use of an ac heterodyne lateral shear interferometer with real-time wavefront correction systems. Appl. Opt. 14, 2622-2626.

Yoder, C. F., 1995. Astrometric and Geodetic Properties of Earth and the Solar System. In: Ahrens, T. J. (Ed.), Global Earth Physics: A Handbook of Physical Constants. p. 1.

Young, A. T., Schorn, R. A., Young, L. D. G., Crisp, D., Jun. 1979. Spectroscopic observations of winds on Venus. I - Technique and data reduction. Icarus 38, 435-450.

Young, L. A., Yelle, R. V., Young, R., Seiff, A., Kirk, D. B., Jan. 2005. Gravity waves in Jupiter's stratosphere, as measured by the Galileo ASI experiment. Icarus173, 185-199. 